\title{
Nanostructured and Heterostructured 2D Materials for Thermoelectrics
}

\author{
Qinyi Li, ${ }^{1,5, *}$ Qing Hao, ${ }^{2, *}$ Tianhui Zhu, ${ }^{3}$ Mona Zebarjadi ${ }^{3,4, *}$ and Koji Takahashi ${ }^{1,5}$
}

\begin{abstract}
The rapid development in the synthesis and device fabrication of two-dimensional (2D) materials provides new opportunities for their wide applications in a variety of fields including thermoelectric energy conversion, thermal management, and thermal logics. As one important research direction, the possibly poor thermoelectric performance of the pristine 2D materials can be dramatically improved with patterned nanostructures, and stacking of different 2Ds to form layered heterostructures, as well as electrostatic gating, which allow the fine-tuning of both the quasi Fermi level and phonon transport. This article reviews the recent advancement in this direction, with an emphasis on both fundamental understanding and practical problems.
\end{abstract}

Keywords: 2D Materials; Thermoelectrics; Nanostructure; Heterostructure.

Received: 3 August 2020; Accepted: 7 September 2020.

Article type: Review article.

\section{Introduction}

Solid-state thermoelectric devices can directly convert heat into electricity or utilize electricity to pump heat. These devices can be utilized to address two significant issues in the development of emerging micro- and nano- scale devices. One issue is how to power the ubiquitous sensors in the future Internet of Things (IoT) society, where the thermoelectric power generator utilizing the Seebeck effect is one of the most promising self-powering technologies to harvest energy from waste heat and the environment. The second is how to dissipate the increasingly high heat flux from a hot spot in the large-scale integration, where a solid-state Peltier cooler can play an irreplaceable role in the efficient heat removal. In the

\footnotetext{
${ }^{I}$ Department of Aeronautics and Astronautics, Kyushu University, Fukuoka 819-0395, Japan.

${ }^{2}$ Department of Aerospace and Mechanical Engineering, University of Arizona, 1130 N Mountain Ave, Tucson, AZ, 85721, USA.

${ }^{3}$ Department of Electrical and Computer Engineering, University of Virginia, Charlottesville, Virginia, 22904, USA.

${ }^{4}$ Department of Materials Science and Engineering, University of Virginia, Charlottesville, Virginia, 22904, USA.

${ }^{5}$ International Institute for Carbon-Neutral Energy Research (WPI-

I2CNER), Kyushu University, Japan.

*E-mail: qinyi.li@aero.kyushu-u.ac.jp (Q.Y. Li), qinghao@ arizona.edu (Q.

Hao),m.zebarjadi@virginia.edu (M. Zebarjadi)
}

context of power generation, the performance of thermoelectric material is evaluated by a well-defined dimensionless property, figure of merit, i.e. $Z T=S^{2} \sigma T /\left(k_{\mathrm{e}}+k_{1}\right)=P F T / k$, where $S=-\Delta V / \Delta T$ is the Seebeck coefficient (thermopower), $\sigma$ the electrical conductivity, $T$ the absolute temperature; $k$ is the thermal conductivity that can be divided into the electronic part, $k_{\mathrm{e}}$, and the lattice part, $k_{1}$; and $P F=S^{2} \sigma$ is defined as the power factor $(P F)$ that evaluates the ability to output a high electric power with an applied temperature difference or oppositely. ${ }^{[1]}$ A good thermoelectric material for power generation requires a high $P F$, and at the same time a low $k$ to minimize the conduction heat leakage and thus maintains the temperature difference. A high $Z T$ is also desired for a Peltier refrigerator to pump heat from a cold source to a hot end. However, both a high $P F$ and a high $k$ are beneficial in the context of thermal management where heat is transferred from a hot spot to a heat sink. ${ }^{[2-4]}$ Note that the product of $P F$ and $T$, i.e. $P F T$, has the same unit as the thermal conductivity of Watt per meter per Kelvin. Thus, PFT represents the ability of active cooling using the Peltier effect, while $k$ represents the ability of passive cooling. ${ }^{[2,5]}$ For either power generation or thermal management, we need to manipulate $S, \sigma$, and $k$ for improved performance, which has been particularly challenging for decades because these transport properties are generally intertwined. 
There are mainly two strategies to enhance the thermoelectric figure of merit: (1) to suppress phonon transport without significantly affecting electron transport, (2) to engineer the band structure for a higher $P F$. It is beneficial to increase the number of modes within the Fermi window near the band edges, which can be realized by reducing the dimensionality. ${ }^{[6-9]}$ A sharp density of states (DOS) profile at the band edge is expected in 2D materials, and superior electron mobility has been observed in many $2 \mathrm{D}$ crystals like graphene and 2D transition metal dichalcogenides (TMDCs), which are the reasons why 2D materials are promising for thermoelectric applications. Another advantage of 2D materials is their environment-friendliness and flexibility. Traditional thermoelectric materials with $Z T>1$ usually contain toxic or rare elements like $\mathrm{Bi}, \mathrm{Te}, \mathrm{Pb}$, and $\mathrm{Ag}$, which have long hindered their large-scale deployment due to the high cost and environmental harm. Besides, these hard materials are not suitable for energy harvesting in small-scale, flexible devices. Therefore, 2D layered materials with nontoxic, low-weight, and flexible features have attracted increasing attention for thermoelectric applications that are integrated into small-scale and flexible devices.

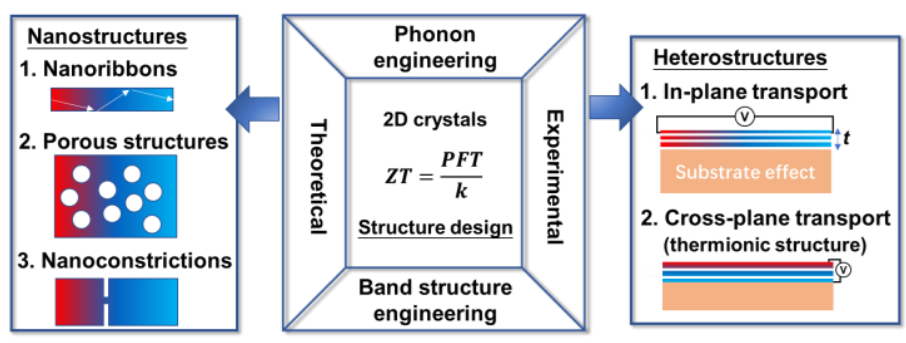

Fig. 1 Concept and outline of this review.

Many 2D materials in the pristine form indeed possess a relatively high $P F$. For pristine graphene without doping, the Fermi level is at the Dirac point or charge neutrality point so that $S$ and $S^{2} \sigma$ are both zero. ${ }^{[27]}$ The sign of the Seebeck coefficient changes from negative to positive across the charge neutrality point, where the majority charge carrier switches from electrons to holes. With an applied gate voltage to optimize the Fermi level, semimetallic graphene exhibits an improved but moderate Seebeck coefficient of about 80 $\mu \mathrm{V} / \mathrm{K} \cdot{ }^{[1,10,11]}$ However, its $P F$ can be up to $10 \mathrm{~mW} / \mathrm{m} \cdot \mathrm{K}^{2}$ due to the extremely high mobility. ${ }^{[10]}$ Semiconducting bilayer $\mathrm{MoS}_{2}$ can exhibit a $P F$ up to $8.5 \mathrm{~mW} / \mathrm{m} \cdot \mathrm{K}^{2}{ }^{[12]}$ about twice that of bulk $\mathrm{Bi}_{2} \mathrm{Te}_{3}$. However, the figure of merit of the existing pristine 2D materials is orders of magnitude lower than commercial thermoelectric materials such as $\mathrm{Bi}_{2} \mathrm{Te}_{3}$ alloys. For example, the $Z T$ values of pristine graphene ${ }^{[10]}$ and $2 \mathrm{D}$ $\mathrm{MoS}_{2}$ are only on the order of $10^{-3}$ and $10^{-2}$, respectively. Therefore, it is desired to independently engineer both electron and phonon transport to improve the thermoelectric performance of 2D materials.

Two approaches can be used to modify the structure of a 2D system and alter the thermoelectric properties accordingly. First, a 2D sheet can be patterned into a nanostructure like a nanoribbon or a porous mesh through lithographical or special synthetic approaches. As pioneered by Hicks and Dresselhaus in $1993,{ }^{[13,14]}$ nanostructures can alter the band structure. In later studies, suppressed phonon transport is often found as another important benefit for nanostructured materials with strong boundary or interface phonon scattering. ${ }^{[15,16]}$ For charge carriers with usually shorter mean free paths (MFPs) than those for phonons, they are less scattered so that bulk-like electrical properties can be preserved. In general, $S, \sigma$, and $k$ can be hopefully decoupled and independently manipulated by careful structure design. Second, different 2D crystals can be stacked layer by layer to establish a van der Waals heterostructure where the band structure as well as phonon transport can be tuned by the van der Waals interfaces, promisingly leading to an enhanced thermoelectric performance. Besides, the thermoelectric properties of 2D crystals can be significantly modified by the type of the substrate, and the number of layers. Up to now, researchers have exfoliated or synthesized a few dozen of 2D crystals, with at least one thousand more still waiting to be discovered. ${ }^{[17-19]}$ Since the nanostructuring and stacking approaches can yield infinite possibilities for constructing a 2D system, the only limitation for tuning the thermoelectric properties is our imagination and a rational approach of the structure design.

In recent years, the thermoelectric studies of 2D materials have been reviewed by several articles from different angles..$^{[1,2,20-27]}$ Some are focused on a particular 2D material, e.g. graphene, and some are focused on particular transport properties, e.g. thermal transport or electrical transport with an emphasis on how to tune the Fermi level. The present review is devoted to the tunability of both electron and phonon transport using the structure manufacturability of 2D materials, including critical reviews of the latest experimental and theoretical progress. We will start from a summary of the thermoelectric properties of pristine 2D materials, and then successively discuss the nanostructured and heterostructured 2D materials for the manipulation of thermoelectric properties and give our perspectives in the last section.

\section{Thermoelectric properties of pristine 2D materials}

In this section, the thermoelectric properties of $2 \mathrm{D}$ materials are reviewed. It should be noted that the possibly low $Z T$ values of these pristine materials may be largely improved with the nanostructuring approach that will be discussed in later sections.

\subsection{Thermoelectric properties of single- and few- layer graphene}

Monolayer graphene is an intrinsic semimetal with a gapless band structure. Several measurements of monolayer graphene supported on $\mathrm{SiO}_{2}$ have confirmed a Seebeck coefficient of about $80 \mu \mathrm{V} / \mathrm{K}$ under an optimized gate voltage to tune the Fermi level ${ }^{[1,10,11,29,30]}$ and an electrical conductivity on the order of $10^{6} \mathrm{~S} / \mathrm{m}$ at room temperature (RT) for high-quality 
samples. ${ }^{[10]} \mathrm{A}$ high $P F$ up to about $10 \mathrm{~mW} / \mathrm{m} \cdot \mathrm{K}^{2}$ was reported for exfoliated single-layer graphene on a suspended $\mathrm{SiO}_{2}$ thin film, ${ }^{[10]}$ which is more than twice that of commercial bulk $\mathrm{Bi}_{2} \mathrm{Te}_{3}$ alloys. However, pristine graphene has an extremely high thermal conductivity of around $3000 \mathrm{~W} / \mathrm{m} \cdot \mathrm{K}$ in the suspended form ${ }^{[10-35]}$ and $600 \mathrm{~W} / \mathrm{m} \cdot \mathrm{K}$ in the $\mathrm{SiO}_{2}$-supported form ${ }^{[10]}$ at RT leading to a very small $Z T$ value on the order of $10^{-3}$. Here, the considerably reduced thermal conductivity of the supported graphene is caused by the suppression of flexural phonons due to the amorphous substrate. Due to its small $Z T$ value, large-area pristine monolayer graphene is not suitable for thermoelectric power generation, but the relatively high $P F$ and remarkably high thermal conductivity can be utilized for efficient active cooling of a hot spot. ${ }^{[2]}$

Bulk graphite is also a semimetal with a high thermal conductivity up to $2000 \mathrm{~W} / \mathrm{m} \cdot \mathrm{K}$. The thermal conductivity of free-standing graphene is larger than that of bulk graphite and increases as the thickness decreases. However, the thermal conductivity of supported graphene is suppressed by the substrate coupling and increases with increasing the layer number. The thermal conductivity of supported layered graphite recovers to the bulk graphite value when the thickness is more than 30 layers. ${ }^{[31]}$

The bilayer and trilayer graphene are intrinsically semimetals but their bandgaps and electronic properties can be widely tuned by a transverse electric field as well as the twist angle, which involves profound physics ${ }^{[36-39]}$ and can be beneficial for enhancing the Seebeck coefficient. The maximum Seebeck coefficient of bilayer graphene without bandgap tuning was measured to be about $100 \mu \mathrm{V} / \mathrm{K}$ at 250 $\mathrm{K},{ }^{[40]}$ while the maximum Seebeck coefficient of bilayer graphene gapped by the electric field was reported to be up to

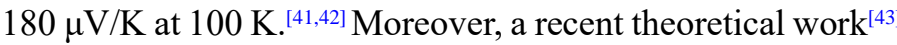
reported a $Z T$ value larger than unity in gapped bilayer graphene at an ultralow temperature of $1 \mathrm{~K}$.

\subsection{Thermoelectric properties of 2D TMDCs}

TMDCs are a family of layered materials with a molecular formula of $\mathrm{MX}_{2}$, where a transitional metal atom, $\mathrm{M}$, is sandwiched by two chalcogen element atoms, $\mathrm{X}$. The 2D forms of semiconducting TMDCs represented by $2 \mathrm{H}$-type $\mathrm{MX}_{2}(\mathrm{M}=\mathrm{Mo}, \mathrm{W} ; \mathrm{X}=\mathrm{S}, \mathrm{Se})$ have potential applications in nanoelectronics and optoelectronics, ${ }^{[44]}$ and many studies have exploited their potentially high thermoelectric performance due to their direct bandgaps and much lower thermal conductivities than that for graphene. ${ }^{[45-71]}$

The thermal conductivities of bulk $\mathrm{MoS}_{2}$ crystals have been measured to be $85-110 \mathrm{~W} / \mathrm{m} \cdot \mathrm{K}$ in the in-plane direction and $2.0 \pm 0.3 \mathrm{~W} / \mathrm{m} \cdot \mathrm{K}$ in the cross-plane direction at RT using the pump-probe metrology based on the magneto-optic Kerr effect. ${ }^{[53]}$ A variable-spot-size time-domain thermoreflectance (TDTR) approach ${ }^{[54]}$ was employed to systematically measure the in-plane and the cross-plane thermal conductivities of bulk $\mathrm{MX}_{2}(\mathrm{M}=\mathrm{Mo}, \mathrm{W} ; \mathrm{X}=\mathrm{S}, \mathrm{Se})$ at $80-300 \mathrm{~K}$. At RT, the in-plane thermal conductivity of the measured TMDCs exhibits the following decreasing order of $\mathrm{WS}_{2}(120 \mathrm{~W} / \mathrm{m} \cdot \mathrm{K})>\mathrm{MoS}_{2}(82$ $\mathrm{W} / \mathrm{m} \cdot \mathrm{K})>\mathrm{WSe}_{2}(42 \mathrm{~W} / \mathrm{m} \cdot \mathrm{K})>\mathrm{MoSe}_{2}(35 \mathrm{~W} / \mathrm{m} \cdot \mathrm{K})$, the trend of which agrees well with first-principles calculations. ${ }^{[56]}$ Another TDTR measurement of disordered, layered $\mathrm{WSe}_{2}$ crystals yielded ultralow out-of-plane thermal conductivity of $0.05 \mathrm{~W} / \mathrm{m} \cdot \mathrm{K}$ at RT. ${ }^{[55]}$ These accurate measurements of the bulk crystals provide a baseline for understanding thermal transport in 2D TMDCs. The thermal conductivities of singleand few-layer TMDCs have been reported by a few Ramanbased ${ }^{[57-63]}$ and micro-bridge-based ${ }^{[64-66]}$ measurements, but the limited data is scattered in a large range (between $\sim 15$ and $\sim 100 \mathrm{~W} / \mathrm{m} \cdot \mathrm{K}$ at $\mathrm{RT}$ for monolayer $\mathrm{MoS}_{2}$ ). Meanwhile, careful MD simulations, ${ }^{[68,50]}$ first-principles ${ }^{[67]}$ and $\mathrm{BTE}^{[69]}$ calculations agreed well on the in-plane thermal conductivities of $2 \mathrm{D} \mathrm{MoS}_{2}$. The values are above $100 \mathrm{~W} / \mathrm{m} \cdot \mathrm{K}$ for the monolayer, decrease with increasing thickness and saturate at the trilayer to be about $80 \mathrm{~W} / \mathrm{m} \cdot \mathrm{K}$. The first-principles calculations $^{[67]}$ reported the RT thermal conductivities of $2 \mathrm{H}$ type TMDC monolayers as $142 \mathrm{~W} / \mathrm{m} \cdot \mathrm{K}$ for $\mathrm{WS}_{2}, 103 \mathrm{~W} / \mathrm{m} \cdot \mathrm{K}$ for $\mathrm{MoS}_{2}, 54 \mathrm{~W} / \mathrm{m} \cdot \mathrm{K}$ for $\mathrm{MoSe}_{2}$, and $53 \mathrm{~W} / \mathrm{m} \cdot \mathrm{K}$ for $\mathrm{WSe}_{2}$. Moreover, this first-principles work found that the 1T-type TMDC monolayers represented by $\mathrm{ZrS}_{2}$ possess a much lower thermal conductivity than the $2 \mathrm{H}$-type monolayers. ${ }^{[6]}$ Besides, Wan and co-workers ${ }^{[71]}$ measured assembled $\mathrm{TiS}_{2}$ layers with organic intercalation, which exhibited an ultralow in-plane thermal conductivity of $0.12 \mathrm{~W} / \mathrm{m} \cdot \mathrm{K}$ that is two orders of magnitude lower than both bulk and monolayer $\mathrm{TiS}_{2}$, along with a high $P F$ of $0.45 \mathrm{~mW} / \mathrm{m} \cdot \mathrm{K}^{2}$, resulting in a $Z T$ value up to 0.28 at $373 \mathrm{~K}$.

As for the $P F$, Wu et al. ${ }^{[48]}$ measured a very large Seebeck coefficient of up to $30 \mathrm{mV} / \mathrm{K}$ at RT in CVD monolayer $\mathrm{MoS}_{2}$, which indicated its promising application in themoelectics. Hippalgaonkar and co-workers ${ }^{[12]}$ measured exfoliated singleto tri- layer $\mathrm{MoS}_{2}$, and obtained a Seebeck coefficient of around $-400 \mu \mathrm{V} / \mathrm{K}$ at a zero gate voltage, while the $P F$ of bilayer $\mathrm{MoS}_{2}$ was as high as $8.5 \mathrm{~mW} / \mathrm{m} \cdot \mathrm{K}^{2}$ at a carrier concentration of $1.06 \times 10^{13} / \mathrm{cm}^{2}$. Another thickness-dependent study on exfoliated $\mathrm{MoS}_{2} / \mathrm{SiO}_{2}$ samples also confirmed that the best $P F$ occurs for bilayer $\mathrm{MoS}_{2}{ }^{[49]} \mathrm{Li}$ and co-workers ${ }^{[51]}$ measured tunable bandgap in monolayer $\mathrm{MoS}_{2}$ that changes with uniaxial strain at a modulation rate of up to $\sim 136 \mathrm{meV} / \%$, thus we may expect an even larger Seebeck coefficient in deformed $\mathrm{MoS}_{2}$ as flexible thermoelectric applications. Besides $\mathrm{MoS}_{2}$, exfoliated $3 \mathrm{~L} \mathrm{WSe}_{2}$ on $\mathrm{SiO}_{2}$ shows a peak Seebeck coefficient of around $200 \mu \mathrm{V} / \mathrm{K}$ for electron conduction and around $250 \mu \mathrm{V} / \mathrm{K}$ for hole conduction at 300 K. ${ }^{[52]}$ The maximum $P F$ reaches $3.7 \mathrm{~mW} / \mathrm{m} \cdot \mathrm{K}^{2}$ for p-type $\mathrm{WSe}_{2}$ and about $3.2 \mathrm{~mW} / \mathrm{m} \cdot \mathrm{K}^{2}$ for $\mathrm{n}$-type, corresponding to electrical conductivities of approximately $3 \times 10^{4}$ and $1 \times 10^{4}$ $\mathrm{S} / \mathrm{m}$, respectively ${ }^{[52]}$ We can combine the separately measured $P F$ and thermal conductivity and estimate $Z T$ to be about 0.02 for pristine $\mathrm{MoS}_{2}$ and $\mathrm{WSe}_{2}$. 


\subsection{Thermoelectric properties of $2 \mathrm{D}$ black phosphorene and layered tin selenide}

2D black phosphorus (or phosphorene) is a narrow-bandgap semiconductor with an orthorhombic puckered honeycomb structure of phosphorus atoms and has attracted great attention due to its high mobility and anisotropic properties. ${ }^{[72]}$ The thermoelectric properties of phosphorene have attracted much attention and relatively high $Z T$ values up to 2 have been theoretically reported. ${ }^{[77-83]}$ Tin selenide $(\mathrm{SnSe})$ layers possess the same puckered lattice structure and anisotropicity as black phosphorus, and the layered bulk SnSe with the 2D nature has been identified as one of the most high-performance thermoelectric materials with an unprecedented $Z T$ value up to 2.6 at $923 \mathrm{~K}^{[84-88]}$ Thus, we can group phosphorene and $\mathrm{SnSe}$ as a family of materials with the same lattice structure that can be very promising candidates as environmentally-friendly $2 \mathrm{D}$ thermoelectric materials.

The thermal conductivity of black phosphorus (BP) nanosheets has been measured by the Raman optothermal method, ${ }^{[73]}$ time-domain thermoreflectance (TDTR) method, ${ }^{[74]}$ two-probe micro-bridge method ${ }^{[75]}$ and four-probe method. ${ }^{[76]}$ Unlike suspended graphene whose thermal conductivity increases with decreasing the thickness, BP nanosheets exhibited a decreasing trend of thermal conductivity in all the three directions as the thickness decreases, ${ }^{[7]]}$ which indicates that monolayer or few-layer BP can have higher $Z T$ values than thick flakes due to both enhanced electrical conductivity and reduced thermal conductivity. For 138-552 nm-thick BP flakes at RT, the crossplane thermal conductivity was measured to be $4.0 \mathrm{~W} / \mathrm{m} \cdot \mathrm{K}$, and the highest in-plane thermal conductivities were 86 and 34 $\mathrm{W} / \mathrm{mK}$ in the zigzag and armchair directions, respectively. ${ }^{[74]}$ The Seebeck coefficient of 10-30 nm thick BP was measured to be up to $\sim 400 \mu \mathrm{V} / \mathrm{K}$ near $300 \mathrm{~K},,^{[82]}$ and the $P F$ of $40 \mathrm{~nm}$ thick BP was measured to be $\sim 4.5 \mu \mathrm{W} / \mathrm{cm} \cdot \mathrm{K}^{2}$ at $210 \mathrm{~K}$. ${ }^{[80]}$ Combining the experimental results of thermal conductivity and $P F$, the $Z T$ value of BP flakes with tens of nanometers thickness can be estimated to be on the order of $10^{-3}$, which indicates much room for the enhancement of thermoelectric performance.As for bulk single-crystal layered SnSe, Zhao and co-workers ${ }^{[84]}$ measured ultralow thermal conductivities of less than $0.4 \mathrm{~W} / \mathrm{m} \cdot \mathrm{K}$ at $923 \mathrm{~K}$, Seebeck coefficients of up to $\sim 550 \mu \mathrm{V} / \mathrm{K}$ at $300 \mathrm{~K}$, and very high $Z T$ values of larger than 2.3 at $723-973 \mathrm{~K}$ even without doping. We can expect an even lower thermal conductivity in single- or few- layer $\mathrm{SnSe}$ because it shares the same lattice structure as BP, and thus 2D $\mathrm{SnSe}$ can be a very promising thermoelectric material.

\subsection{Thermoelectric properties of other 2D sheets}

Researchers have identified some other 2D sheets as potential thermoelectric materials, including 2D tellurium(tellurene), ${ }^{190}$ ${ }^{92]}$ and 2D KAgSe nanosheet ${ }^{[93]}$ etc., the bulk counterparts of which are usually outstanding thermoelectric materials.

Bulk trigonal $\mathrm{Te}$ is a p-type narrow-bandgap semiconductor with the $Z T$ value up to unity around $600 \mathrm{~K}$ and $\sim 0.15$ at $300 \mathrm{~K},{ }^{[89]}$ and some theoretical studies ${ }^{[0,91]}$ indicated an even higher thermoelectric performance for the 2D counterpart of Te due to the quantum confinement. Qiu and co-workers ${ }^{[92]}$ measured the thermoelectric properties of $2 \mathrm{D} \mathrm{Te}$ nanofilms with $\sim 30-\mathrm{nm}$ thickness and $\sim 10 \mu \mathrm{m}$ lateral sizes. With microelectromechanical- systems (MEMS) based resistive thermometers, the RT Seebeck coefficient and $P F$ of the Te nanofilm sample supported on $\mathrm{SiO}_{2}$ were measured to be $413 \mu \mathrm{V} / \mathrm{K}$ and $31.7 \mu \mathrm{W} / \mathrm{cm} \cdot \mathrm{K}^{2}$, respectively. The thermal conductivity of a suspended 2D Te sample was measured by the Raman optothermal method to be $1.5 \mathrm{~W} / \mathrm{m} \cdot \mathrm{K}$, half that of bulk Te. Eventually, the $Z T$ value of $\sim 30-\mathrm{nm}$ thick Te nanofilmswas up to 0.63 at $300 \mathrm{~K}$, four times that of bulk Te.

Theoretically, the thermoelectric properties of atomically thin 2D KAgSe nanosheet were studied by first-principles calculations and the Boltzmann transport equation (BTE), ${ }^{[93]}$ which predicted an ultralow thermal conductivity of 0.03 $\mathrm{W} / \mathrm{m} \cdot \mathrm{K}$ at $700 \mathrm{~K}$ for the trilayer KAgSe and a very high $Z T$ value up to 2.08. The thermoelectric properties of $\mathrm{SnS}_{2}$ nanosheets with a thickness of several tens of nanometers were measured by a MEMS-based device. ${ }^{[95]}$ As the thickness of the $\mathrm{SnS}_{2}$ is decreased, the electrical conductivity is increased while the thermal conductivity is decreased, which leads to a $Z T$ value up to 0.13 at $300 \mathrm{~K}, \sim 1,000$ times that of bulk $\mathrm{SnS}_{2}$. Shimizu and co-workers ${ }^{[96]}$ measured an enhanced Seebeck coefficient in $\mathrm{ZnO}$ thin films with a thickness of several nanometers as compared to bulk $\mathrm{ZnO}$. Hung and co-workers ${ }^{[97]}$ conducted BTE and first-principles calculations for monolayer InSe and found a $P F$ up to $50 \mathrm{~mW} / \mathrm{mK}^{2}$. By using the density functional theory (DFT) and semiclassical BTE calculations, Sharma et al. ${ }^{[98]}$ predicted enhanced $Z T$ in $\mathrm{As}$ and $\mathrm{Sb}$ monolayers as compared to the bulk counterparts. Yu et al. ${ }^{[99]}$ predicted a peak $Z T$ value of 1.84 at $800 \mathrm{~K}$ in single-layer ptype BiOBr. Wang and co-workers ${ }^{[110]}$ studied another $2 \mathrm{D}$ carbon allotrope named graphyne using the first-principles calculations and the nonequilibrium Green's function (NEGF) formalism and obtained a $Z T$ value of 0.16 at RT that is much larger than that of graphene.

\section{Nanostructured 2D materials for thermoelectrics}

As observed in Section 2, some 2D materials like graphene may not be attractive for conventional thermoelectric applications. However, unfavorable properties like an ultrahigh thermal conductivity can be tailored by introducing 2D nanofeatures across the atomic-thick material, e.g., nanoribbons or periodic pores. This Section will summarize the major advancement in this important research direction.

\subsection{Theoretical progress}

To compute the lattice thermal conductivity, two major approaches are employed for 2D materials, namely firstprinciples calculations and molecular dynamics (MD) simulations. The accuracy of MD simulations can be largely improved with first-principles computed interatomic force constants. ${ }^{[100]}$ Atomistic Green's function method ${ }^{[101,159]}$ can 

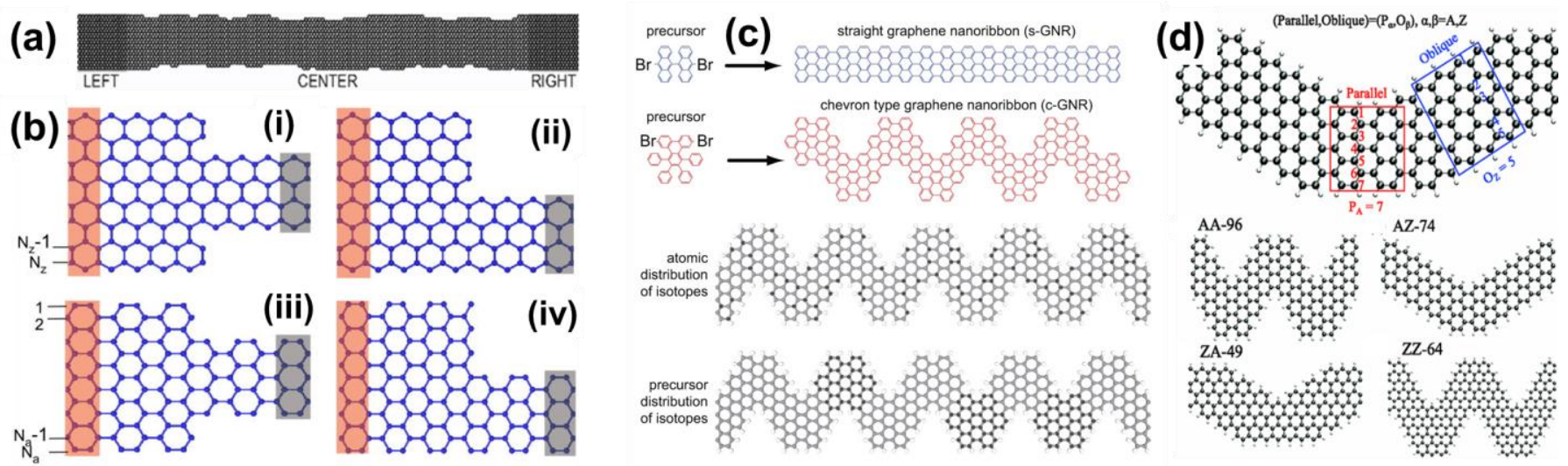

Fig. 2 Graphene nanoribbon (GNR) structures for enhanced thermoelectric performance. (a) An edge disordered zigzag GNR, reproduced with the permission from [119]. Copyright@American Physical Society. (b) T-shaped and L-shaped, zigzag, and armchair GNRs, reproduced with permission from [126]. Copyright@American Institute of Physics. (c) Precursor or isotope-based bottom-up construction of straight and chevron type GNRs, reproduced with permission from [125]. Copyright@Nature Publishing Group. (d) GNRs assembled to various structures, reproduced with permission from [130]. Copyright@American Physical Society.

also be used in certain cases based on the computed phonon transmissivity across a structure. However, inelastic phonon scattering cannot be easily incorporated in this method.

For electrical properties, the required electronic band structures can be computed with first-principles calculations (unit cell of $\sim 100$ atoms or less) or tight binding modeling (unit cell of $\sim 10 \mathrm{~nm}$ sizes or less). For electron conduction, both ballistic and diffuse regimes can be physically formulated by the Landauer approach, ${ }^{[6,7]}$ by which the spectral electrical conductivity per unit energy, $\sigma^{\prime}(E)$, is formulated in terms of the energy $(E)$-dependent transmission of electrons, $\mathcal{T}_{e}(E)$, given by ${ }^{[6]}$

$$
\sigma^{\prime}(E)=\frac{L}{A} \frac{2 e^{2}}{h} \mathcal{T}_{e}(E) M_{e}(E)\left(-\frac{\partial f_{F D}}{\partial E}\right)
$$

where $M_{\mathrm{e}}(E)$ is the energy-dependent number of modes that is proportionally related to the DOS of electrons, $e$ is the electron charge, $f_{\mathrm{FD}}$ is the Fermi-Dirac distribution function, $h$ is the Planck constant, $L$ is the sample length and $A$ is the crosssectional area. The electron transmission function, $\mathcal{T}_{e}(E)$, is related to the energy-dependent MFP, $\lambda(E)$, by $\mathcal{T}_{e}(E)=$ $\lambda(E) /(\lambda(E)+L) \cdot{ }^{[102]}$ The total electrical conductivity is then given by

$$
\sigma=\int \sigma^{\prime}(E) d E
$$

The Seebeck coefficient, $S$, is derived to be: ${ }^{[6]}$

$S=-\frac{1}{e T} \frac{\int\left(E-E_{F}\right) \sigma^{\prime}(E) d E}{\sigma}=-\frac{1}{e T}\left[\frac{\int E \mathcal{T}_{e}(E) M_{e}(E)\left(-\frac{\partial f_{F D}}{\partial E}\right) d E}{\int \mathcal{T}_{e}(E) M_{e}(E)\left(-\frac{\partial f_{F D}}{\partial E}\right) d E}-E_{F}\right]$

where $E_{\mathrm{F}}$ is the Fermi energy. Accordingly, the Seebeck coefficient is determined by the difference between the average carrier energy and the Fermi level. The Seebeck coefficient decreases while the electrical conductivity increases with increasing the Fermi level, and there exists a maximum $P F$ at the optimized Fermi level somewhere near the band edge. Further, the electronic thermal conductivity, $k_{\mathrm{e}}$, is formulated by Equation (4):

$$
k_{e}=\frac{1}{e^{2} T} \int\left(E-E_{F}\right)^{2} \sigma^{\prime}(E) d E-T \sigma S^{2}
$$

and the lattice thermal conductivity, $k_{\mathrm{ph}}$, which dominates thermal transport in semiconductors, can be parallelly formulated by the Landauer approach in terms of the transmission function of phonons. ${ }^{[8]}$ Both electrons and phonons can be scattered by defects, impurities, and boundaries, the effects of which are reflected in the MFPs, or the transmission functions of phonons and electrons in the Landauer formalism.

For materials like graphene and boron nitride, particular attention should also be paid to the hydrodynamics of the phonon transport. ${ }^{[103-106]}$ This occurs when the momentumconserving normal process overshadows the resistive Umklapp process. In contrast with bulk materials, where hydrodynamic phonon transport only occurs at extremely low temperatures, ${ }^{[107]}$ phonon hydrodynamics can be found in some 2D materials well above cryogenic temperatures. Similar phonon hydrodynamics is also claimed for graphite even above $100 \mathrm{~K} \cdot{ }^{[108]}$ In this case, heat can propagate in the form of a damped wave and second sound can be hosted within the material. This interesting phenomenon should also be considered in the thermal design of nanostructured 2D materials, such as graphene nanoribbons. ${ }^{[109]}$

\subsubsection{Nanoribbons}

Nanoribbons are strips of 2D materials with a width of approximately less than $100 \mathrm{~nm}$. Different kinds of nanoribbon structures have been studied for thermoelectric properties, including graphene nanoribbons (GNRs) of various shapes, ${ }^{[118-133]}$ sp3-bonded GNRs, ${ }^{[136]}$ silicene and germanene nanoribbons, ${ }^{[137-139]}$ TMDC nanoribbons, ${ }^{[140-142]}$ phosphorene nanoribbons, ${ }^{[143]}$ and hybrid superlattice nanoribbons. ${ }^{[137,144-150]}$. Table 1 lists the reported $Z T$ values of nanoribbons in the existing theoretical studies, which range from 0.1 to $\sim 6$ at RT. 
Table 1. $Z T$ values reported in theoretical studies of nanoribbons.

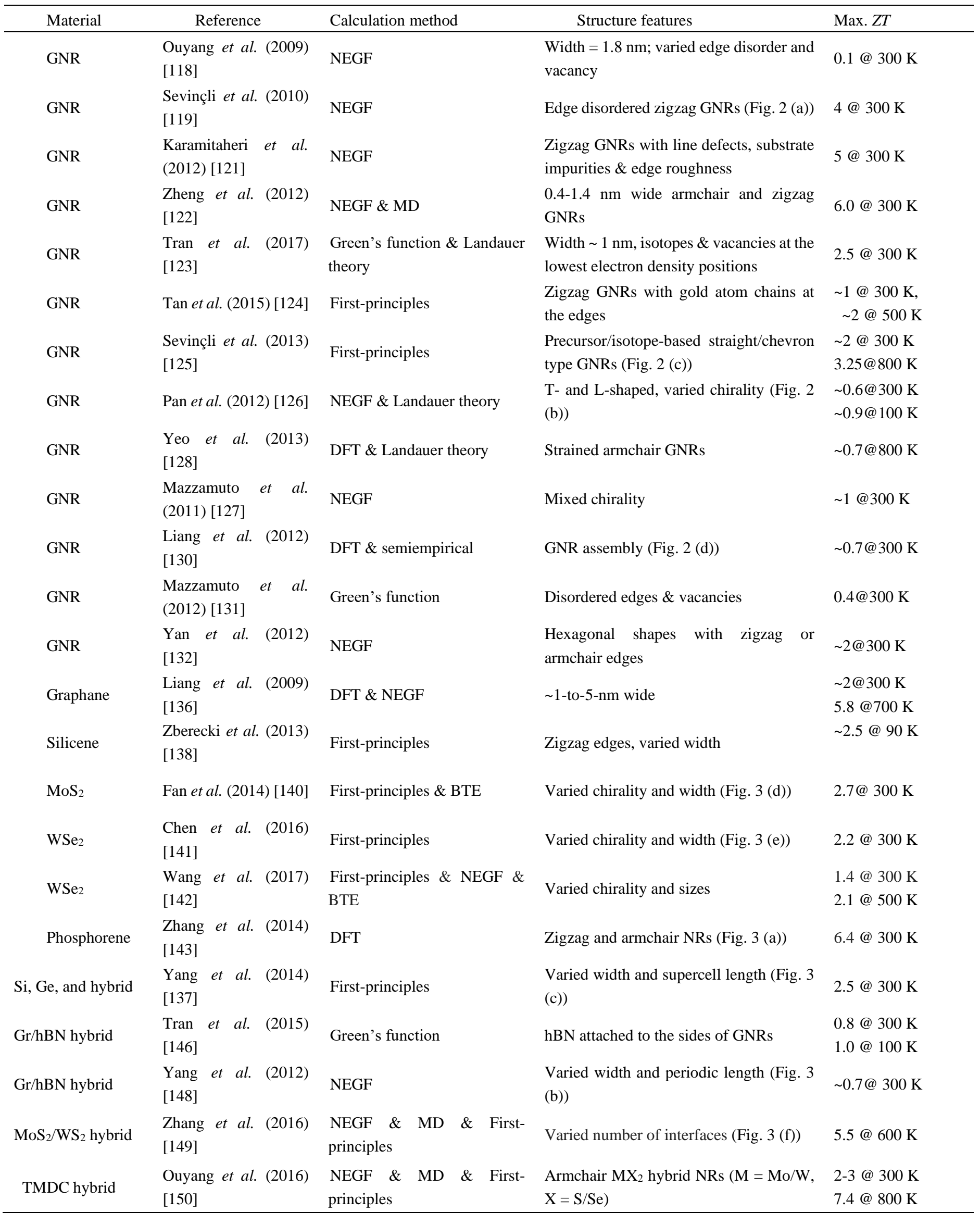

Note: $\mathrm{Gr}=$ graphene 
All these studies reported both enhanced Seebeck coefficient due to the bandgap opening and drastically reduced thermal conductivity as compared with the bulk counterpart, which can be generally explained by phonon scattering with boundaries as well as the ballistic phonon transport because of the nanoscale sizes that are smaller than the phonon MFP. ${ }^{[111-117]}$ For example, the phonon MFP of bulk graphene is up to $\sim 300 \mathrm{~nm}^{[13]}$ and thus the phonon scattering with the rough edges dominates the thermal resistance in a GNR with a width of less than $100 \mathrm{~nm}$, while the ballistic phonon transport can be important in a GNR with a length of less than $300 \mathrm{~nm}$ and reduce the effective thermal conductivity. Generally, the thermal conductivity of nanoribbons with the same edge roughness decreases with decreasing ribbon width. ${ }^{[12]}$ The edge roughness is the other important factor that determines the strength of phonon-edge scattering. Because the zigzag edge is more smooth than the armchair edge, which suggests a weaker phonon scattering with the zigzag edge, it is found that the perfect zigzag GNR has a larger thermal conductivity than the armchair ones with the same width. ${ }^{[115,117]}$ In practice, both experimental and theoretical studies have also demonstrated that the zigzag edges also have a long-termed stability. ${ }^{[114]}$ For instance, the edge of a hole with more armchair configuration initially can be stabilized with more zigzag configuration under electron beam irradiation. Interestingly, phonon scattering gets much stronger at atomically disordered zigzag edges of GNRs (Fig. 2 (a)), which can reduce the thermal conductivity by 10 fold as compared with that of perfect zigzag GNRs. ${ }^{[119]}$ In addition to the phonon-edge scattering, phonon scattering with isotopes ${ }^{[123,124]}$ and bulk defects ${ }^{[18,120,136]}$ has also been explored to further reduce the thermal conductivity of nanoribbons. In the GNRs with non-uniform widths or assembled GNRs (Fig. 2 (b)-(d)), the GNR segments can have different phonon spectra and the phonon transport can be further suppressed due to the thermal boundary resistance between segments as well as the phonon localization. ${ }^{[124-127]}$ Adding nanopillars on both sides of GNRs can significantly reduce the thermal conductivity due to the phonon local resonant hybridization wave effect. ${ }^{[133]}$ Such pillar-modified structures are called "nanophononic metamaterials" and sometimes "phonon resonant structure," which were frist proposed by Nika et al. ${ }^{[134]}$ and Davis et al. ${ }^{[135]}$ Besides, it was found that the tensile strain can reduce the thermal conductivity of GNRs and thus increase the $Z T$ value. ${ }^{[128,129]}$ In the superlattice nanoribbons consisting of hybrid 2D crystals (Fig. 3(b), (c), and (f), ${ }^{[144-150]}$ ), the mechanisms of thermal conductivity reduction include the phonon-interface scattering and interference effects.

Similar to phonon transport, the electron transport can be suppressed by boundary scattering. For the enhancement of $Z T$, the point is how to design a sophisticated nanostructure that can suppress phonon transport much more strongly than electron transport by utilizing the disparate features of phonons and electrons. Especially, defects are inevitable in real samples and it is important to figure out the effects of defects on both electron and phonon transport. Several theoretical studies ${ }^{[18-120]}$ have suggested that disorders at the edges can scatter phonons much more strongly than electrons in GNRs, which can lead to a higher ratio of electrical to thermal conductivities and thus a higher ZT. However, bulk defects inside the GNR suppress electron transport more significantly than phonons, ${ }^{[118,120]}$ which can result in a decreased $Z T$. These results suggest that a clean GNR with rough edges but few bulk defects are preferred for thermoelectric applications. A more recent theoretical study ${ }^{[123]}$ demonstrated that isotopes and vacancies at the lowest electron density positions of GNRs can drastically reduce the thermal conductance without degrading the electrical conductance particularly in the low-energy region, which provides further guidance for sophisticated defect engineering in GNR thermoelectrics.

Table 2. $Z T$ values reported in theoretical studies of $2 \mathrm{D}$ porous structures.

\begin{tabular}{|c|c|c|c|c|}
\hline Material & Reference & Calculation method & Structure features & Max. ZT \\
\hline GAL & Gunst et al. (2011) [157] & Landauer theory & $\begin{array}{l}\text { Hexagonal holes with varied sizes and } \\
\text { edge chiralities }\end{array}$ & $\sim 0.3 @ 300 \mathrm{~K}$ \\
\hline GAL & $\begin{array}{l}\text { Karamitaheri et al. (2011) } \\
{[158]}\end{array}$ & Landauer theory & $\begin{array}{l}\text { Circular, rectangular, hexagonal, and } \\
\text { triangular antidot shapes (Fig. } 4 \text { (c)) }\end{array}$ & $\sim 0.2 @ 300 \mathrm{~K}$ \\
\hline GAL & Yan et al. (2012) [159] & NEGF & 1D antidot arrays (Fig. 4 (a)) & $\sim 1.0 @ 300 \mathrm{~K}$ \\
\hline GAL & Hossain et al. (2015) [160] & DFT \& NEGF & Rectangular hole with varied sizes & $\sim 0.8 @ 300 \mathrm{~K}$ \\
\hline GAL & Chang et al. (2014) [161] & First-principles & $\begin{array}{l}\text { Both heavy adatoms and nanopores (Fig. } 4 \\
\text { (b)) }\end{array}$ & $\sim 3 @ 40 \mathrm{~K}$ \\
\hline Porous silicene & Sadeghi et al. (2015) [162] & DFT & $\begin{array}{l}\text { Varied doping and edge termination (Fig. } \\
4 \text { (d)) }\end{array}$ & $3.5 @ 300 \mathrm{~K}$ \\
\hline
\end{tabular}


(a) Phosphorene nanoribbons

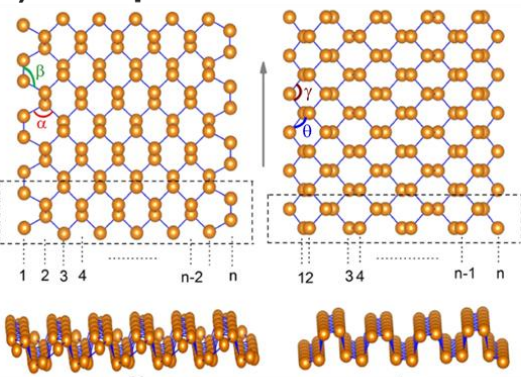

(b) $\mathrm{Gr} / \mathrm{hBN}$ superlattice nanoribbon

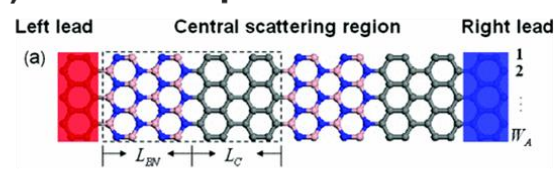

(c) Si/Ge superlattice nanoribbon

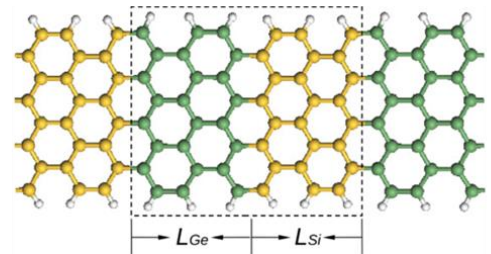

(d) $\mathrm{MoS}_{2}$ nanoribbon

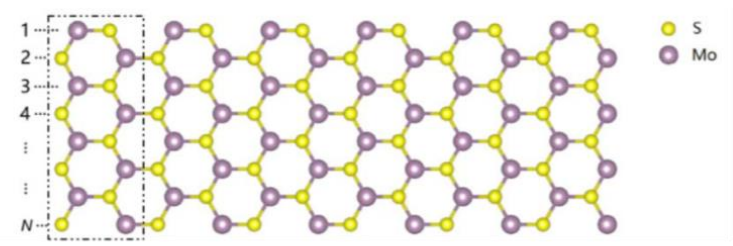

(e) $\mathrm{WSe}_{2}$ nanoribbon

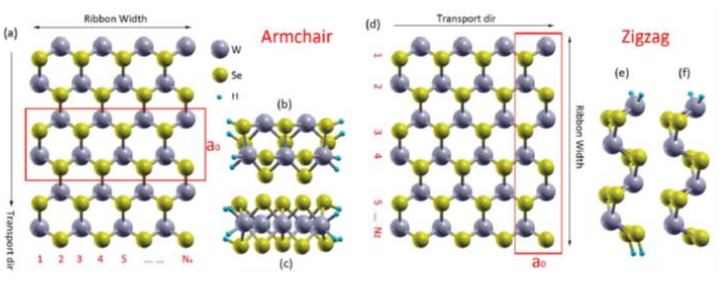

(f) $\mathrm{MoS}_{2} / \mathrm{WS}_{2}$ superlattice nanoribbon

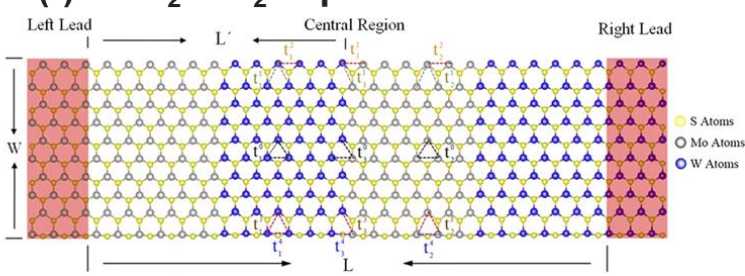

Fig. 3 Various nanoribbons explored for thermoelectric applications. (a) Phosphorene nanoribbons, reproduced with permission from [143]. (b) Graphene/boron nitride superlattice nanoribbons, reproduced with permission from [148]. Copyright@American Physical Society. (c) Si/Ge superlattice nanoribbons, reproduced with permission from [137]. Copyright@American Physical Society. (d) $\mathrm{MoS}_{2}$ nanoribbons, reproduced with permission from [140]. Copyright@American Institute of Physics. (e) $\mathrm{WSe}_{2}$ nanoribbons, with permission from [141]. Copyright@Royal Society of Chemistry. (f) $\mathrm{MoS}_{2} / \mathrm{WS}_{2}$ superlattice nanoribbon (adapted from [149]. Copyright@Nature Publishing Group

\subsubsection{Porous structures}

Another important type of nanostructure is the porous structure where arrays of atomic- to nano- scale holes are introduced inside the 2D lattices. With tunable phonon transport and electron band structures, these 2D porous structures are named as antidot lattices, nanomeshes, or phononic structures. ${ }^{[16,151]}$ Several 2D porous structures have been theoretically investigated for thermal, electronic, and thermoelectric properties, including the most studied graphene antidot lattices (GAL), ${ }^{[151-161]}$ porous silicene, ${ }^{[162]}$ and 2D $\mathrm{MoS}_{2}$ and phosphorene antidot lattices. ${ }^{[163-164]}$ On one hand, phonon transport in these porous structures can be significantly suppressed by boundary scattering and localization of phonons around the pores, as well as wave-like phonon interference in periodic structures. ${ }^{[16,155,156]}$ On the other hand, the electronic band structure can be tailored in well-designed porous structures so that the Seebeck coefficient can be enhanced while the electrical conductivity can remain high. ${ }^{[157-164]} \mathrm{Up}$ to now, several theoretical studies have demonstrated enhanced $Z T$ values in 2D porous structures, as listed in Table 2.

GALs with nanoscale pores have been extensively studied for the electronic properties, ${ }^{[151-154]}$ demonstrating a nonzero band gap around the nanopores. As for thermal transport, Yang and co-workers ${ }^{[155]}$ demonstrated by MD simulations that the thermal conductivity of periodic graphene nanomeshes (GNMs) can be effectively tuned by changing the porosity and period, with the thermal conductivity reduced to 0.1 to 0.01 of that of pristine graphene as the porosity changes from $\sim 21 \%$ to $65 \%$. Feng and Ruan ${ }^{[156]}$ studied thermal transport in GNMs with $5 \mathrm{~nm}$ neck width, 10-20 nm pore sizes, and different pore shapes and arrangements using MD simulations. The thermal conductivity of the GNMs was found to be 3 orders of magnitude lower than that of pristine graphene, and even 200fold lower than that of GNRs with the same neck width, showing great potential for thermoelectric applications. ${ }^{[156]}$ Further, several groups reported simultaneous simulations of phonon and electron transports in various graphene porous structures (or GALs), which yielded reduced thermal conductivity, maintained electrical conductivity, and enhanced Seebeck coefficients. ${ }^{[157-161]}$ As listed in Table 2, the reported $Z T$ values of GALs ranged from 0.2 to $\sim 3$. Especially, Chang and co-workers ${ }^{[161]}$ modified the GAL by adding adatoms, which can enhance the spin-orbit coupling and thus convert bulk grapheneinto a topological insulator with a bandgap and robust helical edge states. While phonon transport was significantly suppressed by the nanopores, the Seebeck coefficient reached up to $1 \mathrm{mV} / \mathrm{K}$ and the maximum $Z T$ value was as high as $\sim 3$ at $40 \mathrm{~K} .{ }^{[161]}$ 
(a) Typical GAL (circular hole)

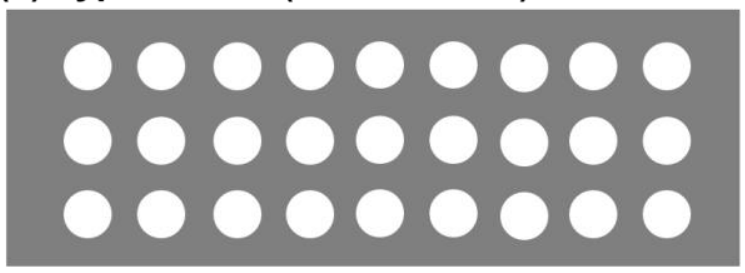

(b) GAL with heavy adatoms

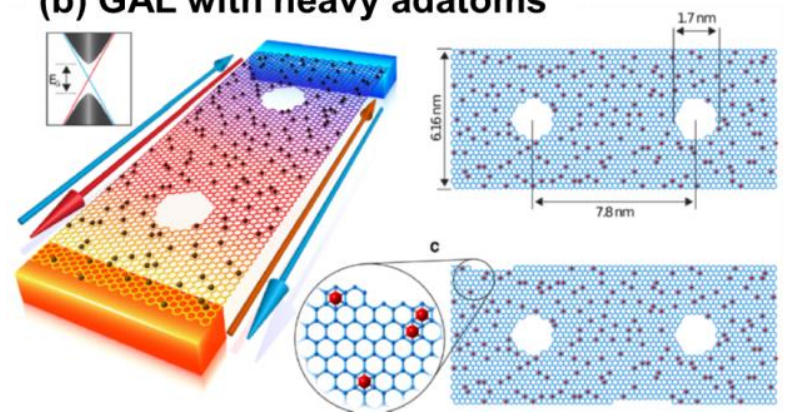

(c) Various GAL

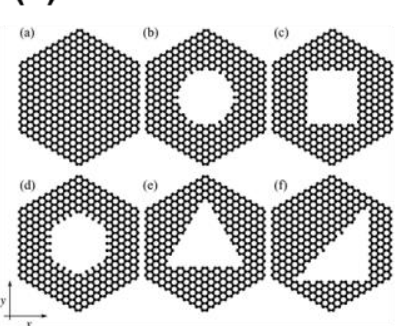

(d) Silicene AL

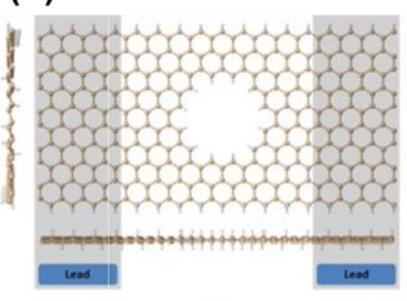

(e) Phosphorene AL

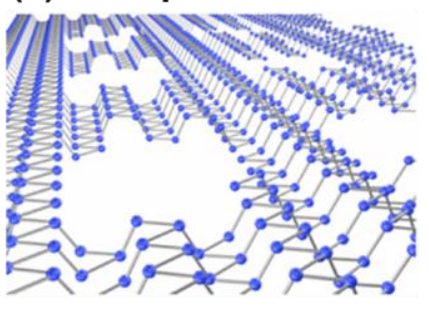

(f) $\mathrm{MoS}_{2} \mathrm{AL}$

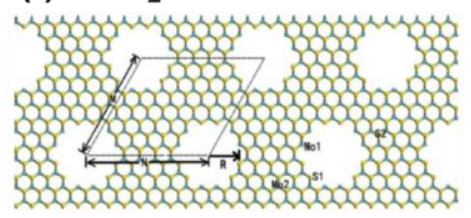

Fig. 4 2D porous structures. (a) Typical GAL with circular holes. (b) GAL with heavy adatoms reproduced with permission from [161]. Copyright@American Chemical Society. (c) GAL with holes of different geometries, reproduced with permission from [158]. Copyright@American Institute of Physics. (d) Silicene antidot lattice, reproduced with permission from [162]. Copyright@Nature Publishing Group. (e) Phosphorene antidot lattice, reproduced with permission from [164]. Copyright@American Chemical Society. (f) $\mathrm{MoS}_{2}$ antidot lattice, reproduced with permission from [163]. Copyright@American Institute of Physics.

Besides graphene porous structures, Sadeghi and coworkers ${ }^{[162]}$ reported enhanced thermoelectric performance of silicene antidot lattices (Fig. 4 (d)), with the Seebeck coefficient up to $500 \mu \mathrm{V} / \mathrm{K}$ and the $Z T$ value as high as 3.5 . Shao at al. ${ }^{[163]}$ reported electronic properties of $\mathrm{MoS}_{2}$ antidot lattices (Fig. 4(f)) using first-principles calculations and found tunable bandgaps with the supercell sizes and the foreign atom adsorption. Cupo and co-workers ${ }^{[164]}$ reported tunable band gaps of phosphorene antidot lattices (Fig. 4 (e)) both theoretically and experimentally. Their DFT calculations revealed that the bandgap scaling can be attributed to quantum confinement effects. ${ }^{[164]}$

\subsubsection{Nanoconstrictions}

The existing studies of 2D materials with circular pores can be further extended to periodic nanoslot patterns as well as other nano constriction structures. Assuming classical phonon/electron size effects, the neck width between adjacent nanopores was suggested as the characteristic length of periodic nanoporous $2 \mathrm{D}$ materials or thin films, ${ }^{[165-167]}$ similar to the role of nanowire diameters in the corresponding phonon transport analysis. As nanoconstrictions, this neck width is actually the minimum feature size within the nanoporous pattern and can yield the lower bound of the actual thermal and electrical conductivities. ${ }^{[16]}$ In contrast with patterns with periodic nanopores, an accurate characteristic length determined by the geometry is available for 2D structures with aligned nanoslots. ${ }^{[168,169]}$ This introduces unique opportunities in the design and optimization of such nanoconstriction structures for thermoelectric applications.

One important aspect of nanoslot-patterned thin films is that phonons with MFPs much longer than the neck width $w$ between adjacent nanoslots will travel ballistically away from the neck, leading to strongly suppressed thermal transport and an associated ballistic thermal resistance. ${ }^{[168,170]}$ If charge carriers in general have a much shorter MFP than phonons, bulk-like electrical properties can be preserved and thus the overall $Z T$ can be enhanced. This $Z T$ enhancement is demonstrated in nanoslot-patterned Si thin films ${ }^{[169]}$ using well-designed nanoslot patterns to adjust the characteristic length. In addition to the $Z T$ enhancement, nanoslot-patterned thin films or 2D materials can also have tunable thermal anisotropy that can be important to thermal management. ${ }^{[171]}$

\subsubsection{Machine learning approaches}

As suggested in the previous sections, there are infinite possibilities for the nanostructure patterns of 2D materials, and hence the case-by-case enumerative studies towards an optimized structure for thermoelectric applications are awfully expensive, inefficient, and often unsuccessful. To address this issue, the machine learning techniques or materials informatics have recently attracted growing interests in the field of thermal transport and thermoelectric studies. ${ }^{[172-176]}$ Combining the limited data accumulated from simulations and experiments with the data-driven machine learning or artificial intelligence (AI) approaches can greatly accelerate the discovery of high-performance thermoelectric structures. Especially, some machine learning algorithms have been utilized to optimize 2D nanostructures for the target properties. Yamawaki and co-workers ${ }^{[172]}$ combined phonon and electron transport calculations of graphene nanostructures with Bayesian optimization, and successfully discovered certain 
structure configurations that can comprehensively optimize the thermal and electrical properties towards an enhanced thermoelectric figure of merit. Two kinds of well-defined graphene nanostructures were investigated for the optimization (Fig. 5): (1) the distribution of vacancy defects in the graphene ribbon, and (2) one-dimensional configuration of the antidot lattice unit and pristine unit. The optimized antidot structure exhibited the highest $Z T$ value that is 11 times that of the pristine GNR and more than five times that was achieved by random search, which was attributed to the zigzag edge states and the unique aperiodic nanostructure.

A recent work by Wei et al. ${ }^{[173]}$ adopted a two-step search process coupled with the genetic algorithm to answer whether the thermal conductivity of GALs with periodically distributed holes is always higher than that of GALs with randomly distributed holes. Around these random holes, the phonon Anderson localization is believed to largely suppress phonon transport. Unexpectedly, Wei and co-workers discovered certain patterns of nanopore distributions with an enhanced thermal conductivity as compared to that of periodic pore distribution, which was never found during the manual search. The unexpected thermal conductivity enhancement was attributed to the shape factor and channel factor in these unusual structures that dominate over the phonon localization. This study demonstrated the possibilities and the complexity of the structure-property relationship in 2D nanostructures, which can stimulate further machine-learning based optimization and provide a guidance for the rational design of two-dimensional structure patterns for enhanced thermoelectric performance.

\subsection{Experimental progress}

This section reviews the progress in experimental studies of

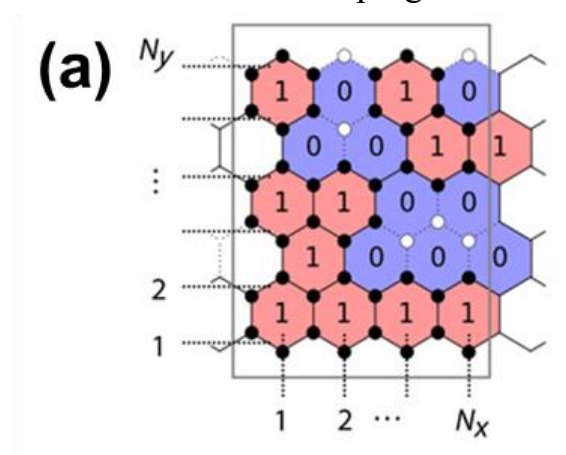

(b)
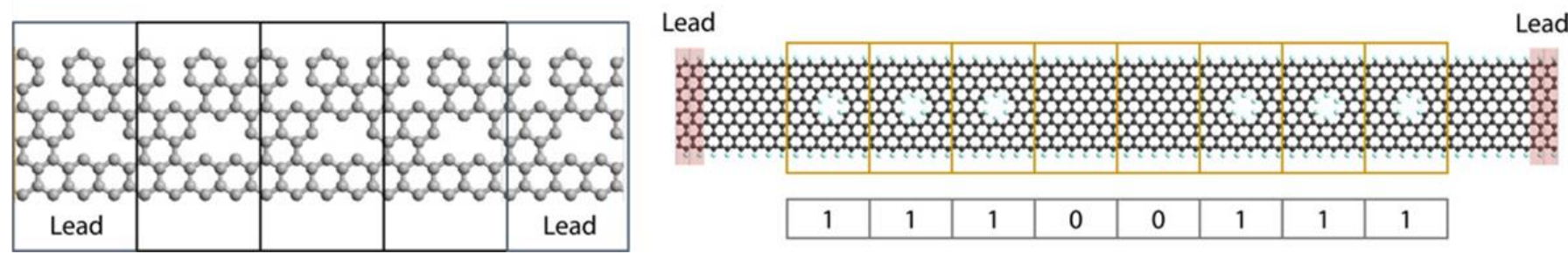

Fig. 5 Bayesian optimization for enhanced $Z T$ of graphene nanostructures. (a) Optimization of the vacancy distribution (Model A). (b) Optimization of the arrangement of the pristine and antidot GNR cells (Model B). The descriptors, 0 and 1, respectively describe the defective and complete hexagonal lattices for Model A, and the pristine and antidot GNR cells for Model B, reproduced with permission from [172]. Copyright@American Association for the Advancement of Science. 

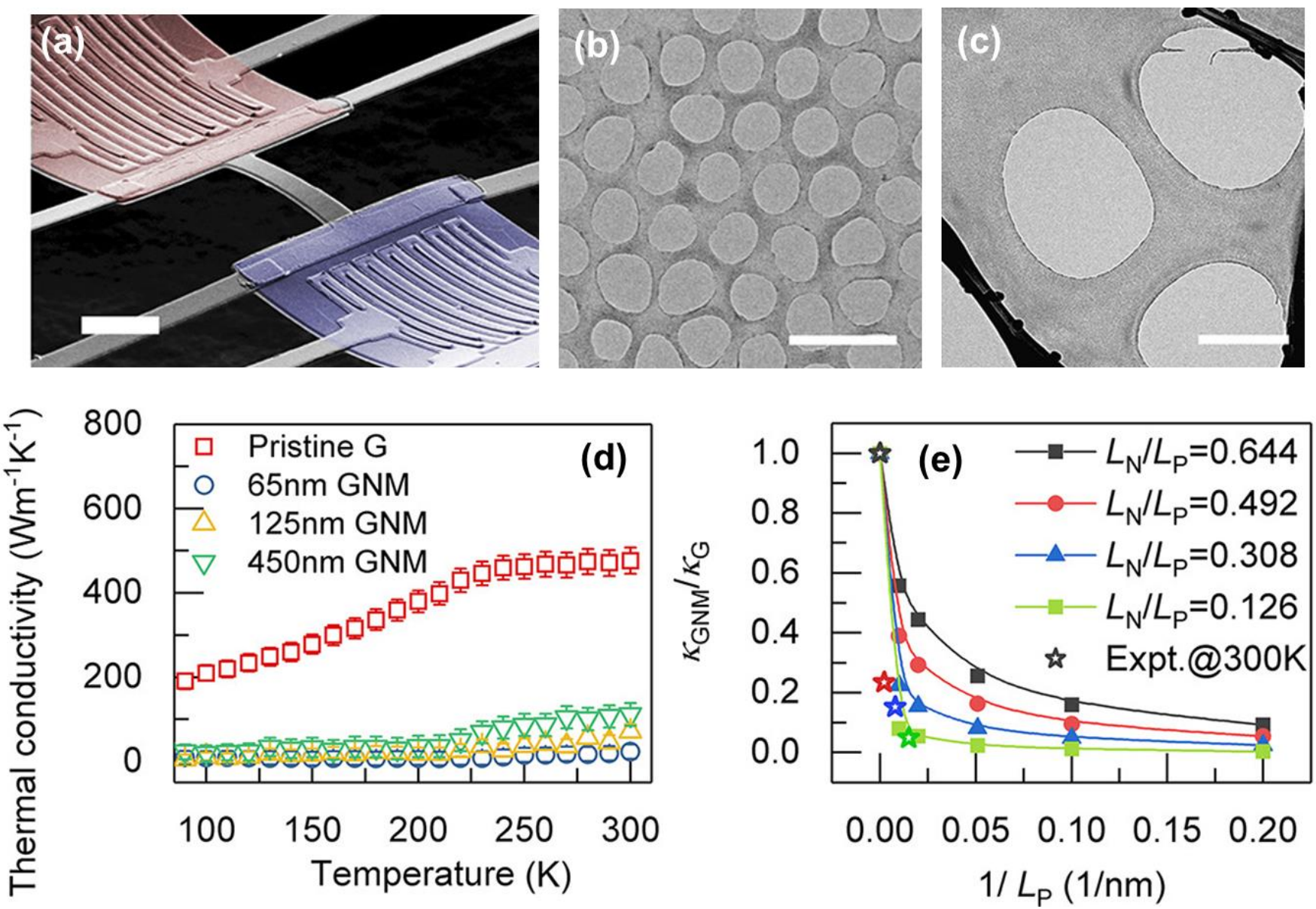

Fig. 6 Thermal transport measurement of GNM supported on $\mathrm{SiO}_{2}$. (a) SEM image of the thermal bridge device (scale bar: $10 \mu \mathrm{m}$ ). (b) TEM image of $125 \mathrm{~nm}$ pitch GNM (scale bar: $200 \mathrm{~nm}$ ). (c) TEM image of $450 \mathrm{~nm}$ pitch GNM (scale bar: $400 \mathrm{~nm}$ ). (d) Measured thermal conductivity of pristine graphene and GNMs with different pitch values. (e) MD simulation results of the normalized thermal conductivity for GNMs with various pitch values $\left(L_{\mathrm{P}}\right)$ and neck widths $\left(L_{\mathrm{N}}\right)$ plotted with the experimental results at $300 \mathrm{~K}$, reproduced with permission from [202]. Copyright@American Chemical Society.

the measured nanostructure. The bridging technique has been used for supported graphene nanoribbons ${ }^{[211]}$ and suspended graphene. ${ }^{[189,190]}$ However, it is not yet implemented for suspended nanoporous structures due to their fragile nature. Metal deposition is often used to form good thermal/electrical contacts between the sample and the suspended device. Noncontact Raman thermometry can also be used to extract the inplane thermal conductivity of a suspended 2D material. The model can be either based on a self-heated suspended strip ${ }^{[191]}$ or laser-heated sample with circular temperature profiles from the laser spot. ${ }^{[192,193,34,213]}$ Besides the less accurate temperature reading of Raman thermometry, an accurate thermal analysis may also involve the strong thermal nonequilibrium between electrons, optical phonons and acoustic phonons ${ }^{[193,206]}$ in laserheated 2D materials.

Keeping these in mind, reliable and systematic thermoelectric measurements should be carried out with more advanced nanofabrication techniques to control the patterns at the atomic level. Nevertheless, the experimental results in this section are still critical and some of the materials can be useful in applications already.

\subsubsection{Porous structures}

Despite many theoretical studies, extremely limited experiments have been carried out to demonstrate the predicted thermoelectric performance of 2D nanostructures due to the challenges of both sample fabrication and thermal measurements at the nanoscale.

The fabrication of 2D porous structures can be classified into three categories: (1) chemical etching or plasma treatment to introduce random and uncontrollable holes or defects; ${ }^{[182,201]}$ (2) helium or gallium ion-beam based direct drilling of holes in the 2D material; ${ }^{[199,200]}$ and (3) mask-assisted etching methods, which include electron beam lithography, ${ }^{[164,196]}$ a soft block-polymer-template assisted method, ${ }^{[195]}$ and a hard template (anodic aluminum oxide thin films) assisted method. ${ }^{[202]}$ The electron beam lithography-based patterning can offer the most accurate and large-scale control of the nanostructure, with the smallest pore diameter around 15 nm. ${ }^{[196]}$ The helium ion beam-based milling yielded the smallest pore size of approximately $6 \mathrm{~nm}$ in diameter. ${ }^{[201]}$

Xiao and co-workers ${ }^{[182]}$ treated few-layer graphene (FLG) with oxygen plasma for 10-20 seconds to introduce atomic- 
scale holes (defects). The Seebeck coefficient of the plasmatreated FLG was measured to be as high as $700 \mu \mathrm{V} / \mathrm{K}$ at 575 $\mathrm{K}$, which was attributed to the bandgap opening induced by the local holes or defects. ${ }^{[182]}$ The electrical conductivity of the randomly etched FLG was $\sim 10^{4} \mathrm{~S} / \mathrm{m}$, which is 2 orders of magnitude lower than high-quality samples, and the $P F$ was $4.5 \mathrm{~mW} / \mathrm{m} \cdot \mathrm{K}^{2}$, only about half that of high-quality pristine graphene. Such plasma treatment can not be used for singlelayer graphene that can be easily destroyed by plasma etching. ${ }^{[182]} \mathrm{A}$ similar random chemical etching was recently adopted by Kang et al. ${ }^{[201]}$ to introduce random holes in $7 \mathrm{~nm}$ thick $\mathrm{MoS}_{2}$ thin films, the in-plane Seebeck coefficient of which was measured to be up to $\sim 742 \mu \mathrm{V} / \mathrm{K}$.

Using the block copolymer self-assembly method, $\mathrm{Oh}$ and co-workers ${ }^{[195]}$ fabricated mono- and bi-layer GNMs with irregular holes. The hole diameter in the nanomeshes was about $20-40 \mathrm{~nm}$, and the average neck widths were $8-21 \mathrm{~nm}$. They measured the Seebeck coefficient and electrical conductivity of $\mathrm{SiO}_{2}$-supported samples by a commercial instrument (TEP-600, Seepel Instrument, Korea), and measured the effective thermal conductivity of a different suspended porous graphene sample by the Raman optothermal method. Note that the Raman-based measurement here can involve large uncertainties due to several factors, including the
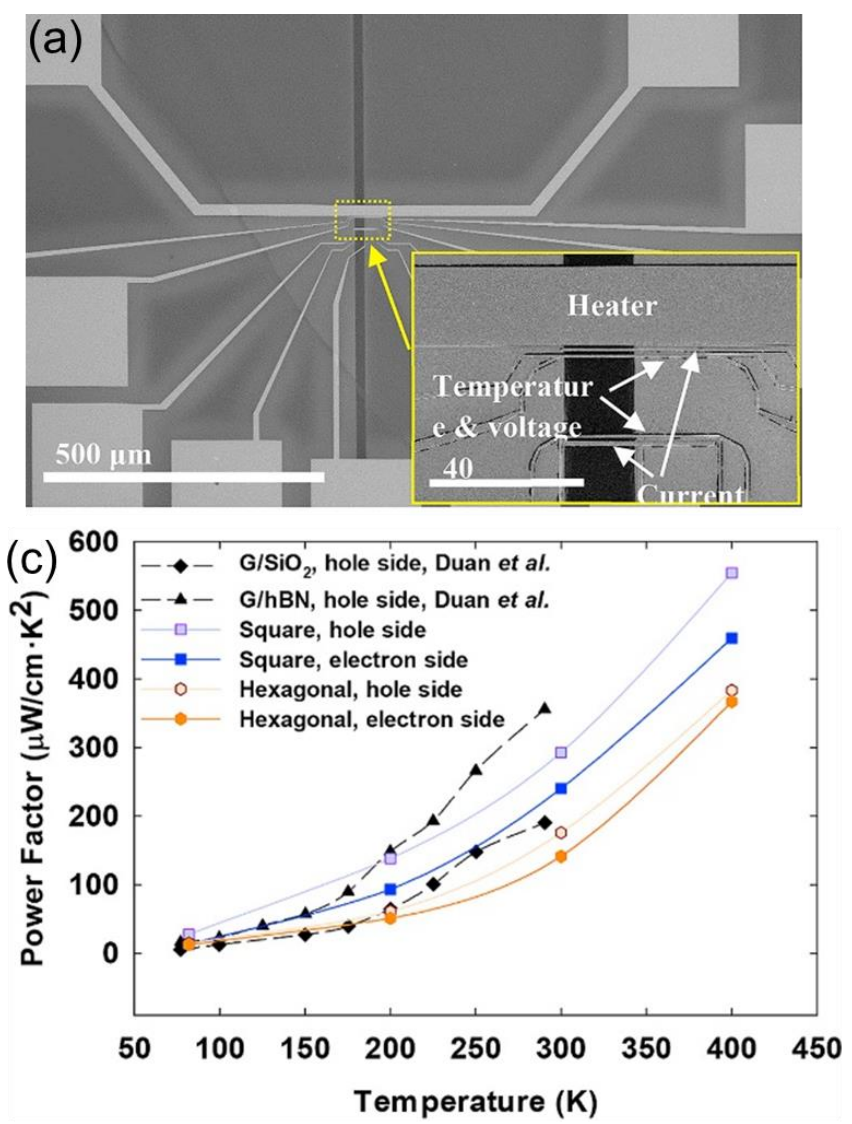

unknown laser absorption and phonon nonequilibrium as well as the relatively low sensitivity for temperature detection, ${ }^{[203-206]}$ which we will discuss in more detail later. The bilayer GNM with $8 \mathrm{~nm}$ neck width exhibited the lowest thermal conductivity of about $78 \mathrm{~W} / \mathrm{mK}$ around $350 \mathrm{~K}$ due to the phonon scattering at the neck edge, and the highest Seebeck coefficient of $-190 \pm 80 \mu \mathrm{V} / \mathrm{K}$ at $300 \mathrm{~K}$ and $-520 \pm$ $92 \mu \mathrm{V} / \mathrm{K}$ at $520 \mathrm{~K}$, which can be explained by the bandgap opening. However, the electrical conductivity was significantly reduced by 2 orders of magnitude to be around 2 $\times 10^{4} \mathrm{~S} / \mathrm{m}$, which should be originated from the defects and contamination introduced in the nanofabrication processes. Oh and co-workers achieved a $P F$ of about $8.2 \mathrm{~mW} / \mathrm{m} \cdot \mathrm{K}^{2}$ at $520 \mathrm{~K}$ in the bilayer nanomesh with $8 \mathrm{~nm}$ neck width due to the superior Seebeck coefficient, but the $Z T$ of all the measured nanostructures were even decreased by $1-2$ orders of magnitude as compared to large-area graphene because the electrical conductivity was not maintained high enough.

Although this work provided the first experimentally measured $Z T$ for GALs, the thermal contact between the sample and the thermal probes may largely affect Seebeck coefficient measurements, where the measured temperature difference can be larger than that "felt" by the sample. Some $P F$ s given by $\mathrm{Oh}$ and co-workers ${ }^{[195]}$ were suspected to be an
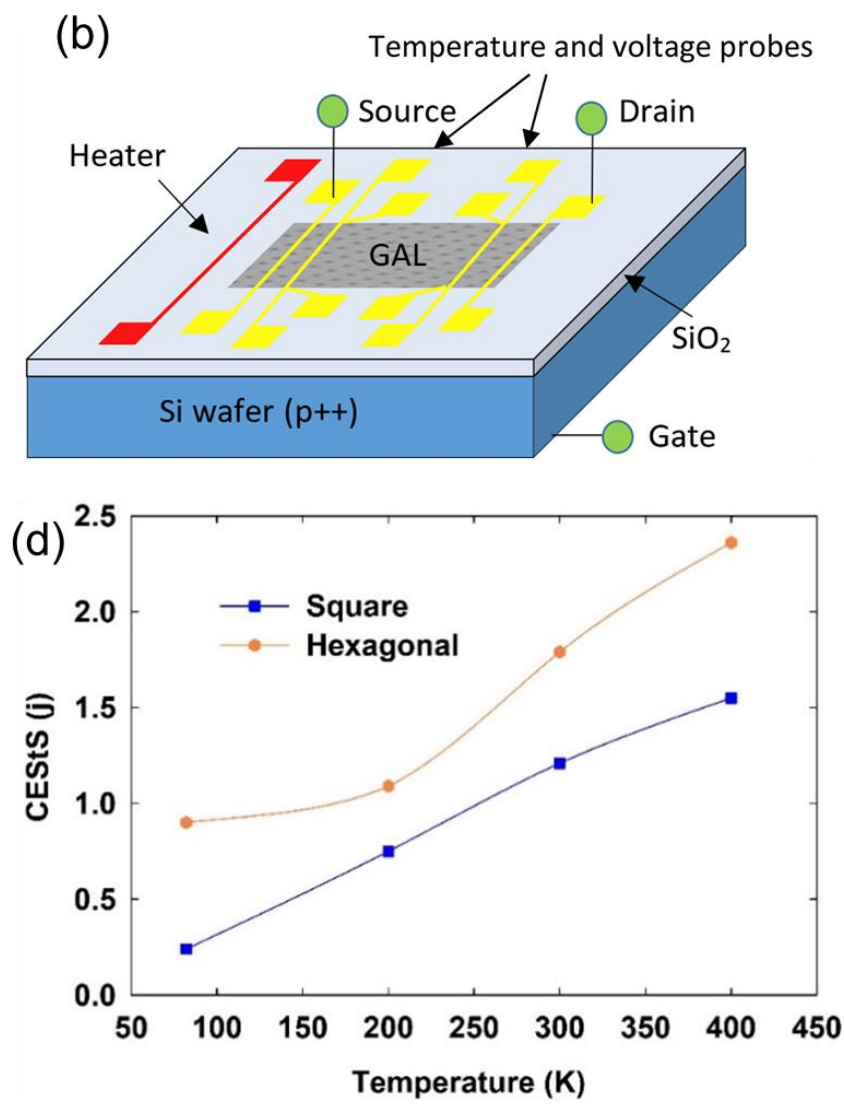

Fig. 7 The enhanced $P F$ of GALs and the detection of major charge-carrier scattering mechanism. (a) SEM image of the test device. (b) Schematic of the measurement principles. (c) $P F$ s of different samples as a function of temperature. (d) The CEStS (j) of GALs with a square array and a hexagonal array of nanopores, reproduced with permission from [196]. Copyright@Elsevier Ltd. 
underestimate of the actual values. On the other hand, their thermal measurements used a Raman laser as a heat source and read the temperature from the Raman peak shift. The laser light was focused on a GAL suspended across a membrane hole. The laser spot acted as a heating source, whereas the membrane was the heat sink to "drain" the heat from the suspended sample. The effective thermal conductivity of a GAL was thus obtained from 2D heat conduction analysis. One challenge was how to determine the actual laser power absorbed by a sample. In the work by $\mathrm{Oh}$ et al., the possible light reflection by the GAL was neglected and the laser power not transmitted through a sample was considered to be absorbed. In addition, the in-air measurements were affected by convection and air conduction around the sample, which is usually hard to be estimated. More accurate measurements also require a better understanding of light absorption by graphene, i.e., the strong thermal nonequilibrium between electrons, optical phonons and acoustic phonons. ${ }^{[193,206]}$

Xiong et al. ${ }^{[202]}$ fabricated GNM supported on $\mathrm{SiO}_{2}$ using a hard-template-assisted etching method and measured the thermal conductivity using the thermal bridge method (Fig. 6). A piece of anodic aluminum oxide thin film with quasihexagonally packed through holes served as a hard mask for the plasma etching of CVD graphene, which can avoid the polymer residue that is usually inevitable with the soft polymer mask. The thermal conductivity of $65 \mathrm{~nm}$ pitch sized GNM was measured to be as low as $\sim 23 \mathrm{~W} / \mathrm{m} \cdot \mathrm{K}$, which can be explained by phonon backscattering at the hole edges as suggested by MD simulations. Using a different line-heater device, they obtained the Seebeck coefficient and electrical conductivity of a $65 \mathrm{~nm}$-pitch GNM sample supported on the substrate, which yielded a power factor of about $2 \mu \mathrm{V} / \mathrm{K}^{2} \cdot \mathrm{cm}$, almost unchanged with the gate voltage, and thus the $Z T$ was estimated to be on the order of $10^{-3}$ at $300 \mathrm{~K}$.

$\mathrm{Xu}$ and co-workers ${ }^{[196]}$ fabricated GALs by electron beam lithography and reactive ion etching. To minimize the measurement errors, deposited metallic electrical/thermal probes were used to ensure good thermal/electrical contacts. The square and hexagonal patterns were compared for their electrical properties. The $P F$ of the square pattern exceeded that of the hexagonal pattern under a gate voltage that maximized $|S|$ (Fig. 7 (c)). At $400 \mathrm{~K}$, a remarkable $P F$ of 554 $\mu \mathrm{W} / \mathrm{cm} \cdot \mathrm{K}^{2}$ was achieved in the p-type GAL with a square pattern, which was far beyond the best $P F \mathrm{~S}$ of bulk thermoelectric materials. One innovation of this work was to better analyze the maximum Seebeck coefficient under an applied gate voltage, from which the carriers' energy sensitivity $(j)$ to scattering $(\mathrm{CEStS})$ was extracted to better understand the scattering mechanisms of charge carriers. In physics, this CEStS is defined as $j=d[\ln (\tau)] / d[\ln \varepsilon]$, where $\tau$ is energy-depdent relaxation time, $\varepsilon$ is the carrier's energy referring to the edge of the corresponding valley. The extracted $j$ in Fig. 7(d) reflects a statistical measure of the average values of $j$ for all existing scattering mechanisms. Because the divergence between electrons and holes is within $10 \%$, the averaged $j$ value is plotted here. Here the positive $j$ value indicates increased importance of the long-range scattering sources at elevated temperatues. This new technique can be applied to general 2D materials to better understand their electron transport processes.

Due to the experimental complexity, the pore-edge configuration (e.g. armchair or zigzag) was not checked in all measured samples. The pore sizes were also significantly larger than those used in computations. In this aspect, highquality samples may be prepared by the direct growth of a nanoporous pattern with $\mathrm{SiO}_{2}$ pillar as the mask that can be removed with hydrofluoric acid etching later. A similar study can be found for the direct growth of nanoporous $\operatorname{In}_{0.1} \mathrm{Ga}_{0.9} \mathrm{~N}$ films for thermal studies. ${ }^{[197]}$ A more perfect structure, with an ordered inclusion of uniform holes and nitrogen atoms, was directly synthesized. Examples are $\mathrm{C}_{2} \mathrm{~N}-\mathrm{h} 2 \mathrm{D}$ crystals ${ }^{[250]}$ and polyaniline $\left(\mathrm{C}_{3} \mathrm{~N}\right)$ with six nitrogen atoms periodically surrounding a phenyl ring. ${ }^{[251]}$ Phonon studies are also carried out on monolayer $\mathrm{C}_{3} \mathrm{~N}$ structure with a periodic array of zigzag holes. ${ }^{[198]}$

\subsubsection{Graphene nanoribbons}

Han and co-workers ${ }^{[210]}$ first experimentally demonstrated the bandgap opening in lithographically patterned GNRs in 2007. The bandgap increased from 3 to $300 \mathrm{meV}$ as the GNR width decreased from 90 to $15 \mathrm{~nm},{ }^{[210]}$ thus we can expect an enhanced Seebeck coefficient in GNRs with a width of several tens of nanometers. Recent experimental progress has also confirmed a drastic reduction of thermal conductivity in both $\mathrm{SiO}_{2}$-supported and suspended GNRs. Bae and co-workers ${ }^{[211]}$ patterned exfoliated graphene sheets into arrays of GNRs with 260 nm length and 45-130 nm widths using electron beam lithography and deposited a hearer and a sensor on top for the thermal conductivity measurement of $\mathrm{SiO}_{2}$-supported GNRs. The thermal conductivity exhibited a clear width $(W)$ dependence that scales as $k \sim W^{1.8 \pm 0.3}$. The room-temperature $k$ of 65-nm wide supported GNRs was suppressed to about 100 $\mathrm{W} / \mathrm{m} \cdot \mathrm{K}$, about one-sixth of large-area supported graphene. ${ }^{[10]}$ This reduction of $k$ was mainly attributed to the strong scattering between phonons and the plasma-etched rough ribbon edges.

Also using the lithographical patterning and plasma etching followed by substrate etching, $\mathrm{Li}$ and co-workers ${ }^{[208]}$ measured the thermal and electrical conductivities of an individual suspended GNR with $169 \mathrm{~nm}$ width and $846 \mathrm{~nm}$ length. The RT thermal and electrical conductivities of the 169nm-wide suspended GNR were about $350 \mathrm{~W} / \mathrm{m} \cdot \mathrm{K}$ and $9 \times 10^{5}$ $\mathrm{S} / \mathrm{m}$, respectively. This thermal conductivity was about $15 \%$ of that of large-area suspended graphene, which can be explained by phonon-edge scattering, but the electrical conductivity was also largely reduced as compared to pristine graphene because of the inevitable defects and contaminations from the lithographical processes. The $Z T$ value of this sample could be estimated to be on the order of $10^{-3}$ by assuming theSeebeck coefficient the same as that of large-area graphene. 
Later, using MEMS fabricated metallic heater and sensors, Hossain and co-workers ${ }^{[209]}$ measured the Seebeck coefficient of graphene sheets and a patterned individual 50-nm-wide GNR supported on $\mathrm{SiO}_{2}$. The Seebeck coefficient was measured to be enhanced from $\sim 10 \mu \mathrm{V} / \mathrm{K}$ for the graphene sheet to $\sim 30 \mu \mathrm{V} / \mathrm{K}$ for the GNR, which demonstrated the nanostructure effect on the Seebeck coefficient although both were quite low values due to the sample quality.
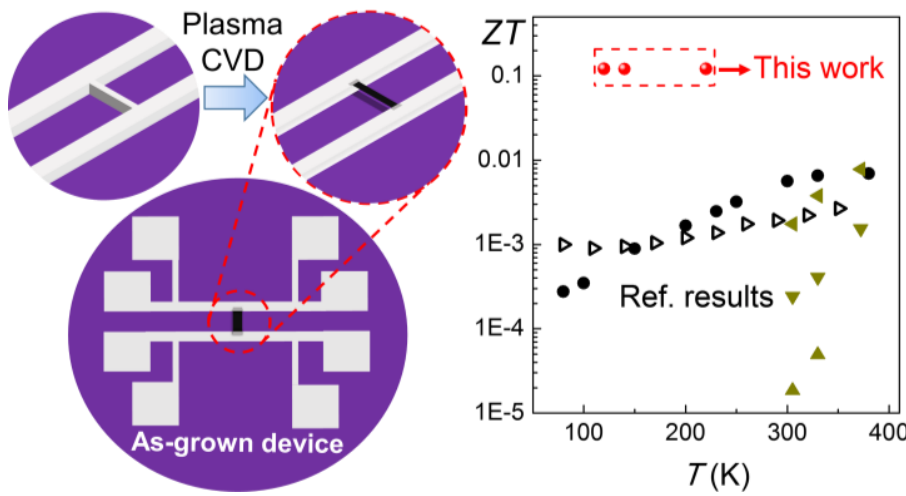

Fig. 8 Record-high $Z T$ measured in as-grown 40-nm-wide suspended GNRs, reproduced with permission from [207]. Copyright@American Chemical Society.
A recent work by Li et al. ${ }^{[207]}$ finally demonstrated the enhancement of $Z T$ of graphene by several orders of magnitude (Fig. 8). They designed a one-process approach to fabricate suspended high-quality GNRs along with an 8terminal test device. Instead of lithographical patterning, the suspended GNRs were directly synthesized by a method called plasma chemical vapor deposition. In this synthesis method, a nanoscale Ni thin film bridging the Ni heater and sensor acted as the catalyst and melted during the synthesis, leaving the above GNR free-standing. The as-grown process ensured a low number of defects and relatively smooth GNR edges, which is important for maintaining high electrical conductivity. The 8-terminal device allowed simultaneous measurements of thermal and electrical conductivities and the Seebeck coefficient. The 40-nm-wide and 250 -nm-long samples exhibited record-high ratios of electrical conductivity to thermal conductivity for graphene that were 1-2 orders of magnitude higher than those previously reported. Detailed phonon BTE simulations revealed that the reduction of thermal conductivity mainly resulted from quasi-ballistic phonon transport, and the significant enhancement of $\sigma / k$ is attributed to disparate electron and phonon MFPs and the defect-free samples. Furthermore, the Seebeck coefficient was
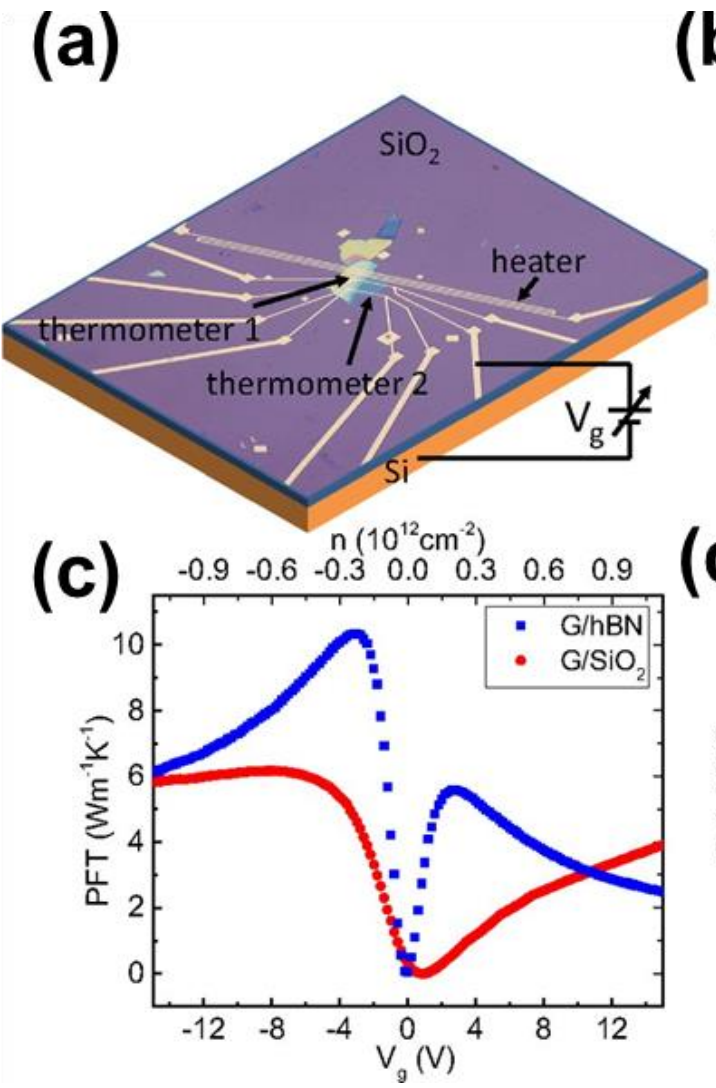

(b)

$\mathrm{n}\left(10^{12} \mathrm{~cm}^{-2}\right)$

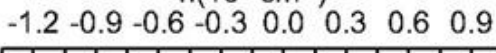

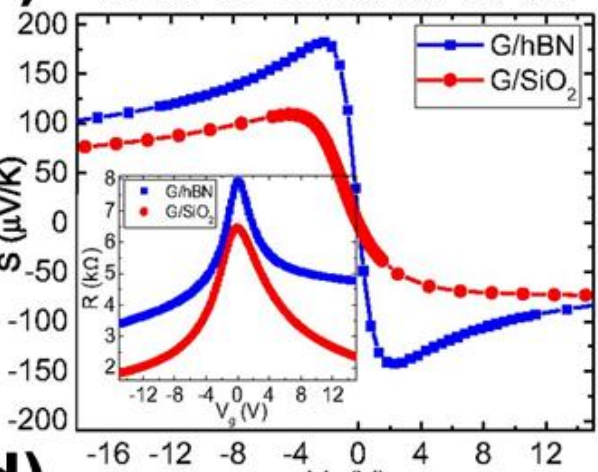

(d)

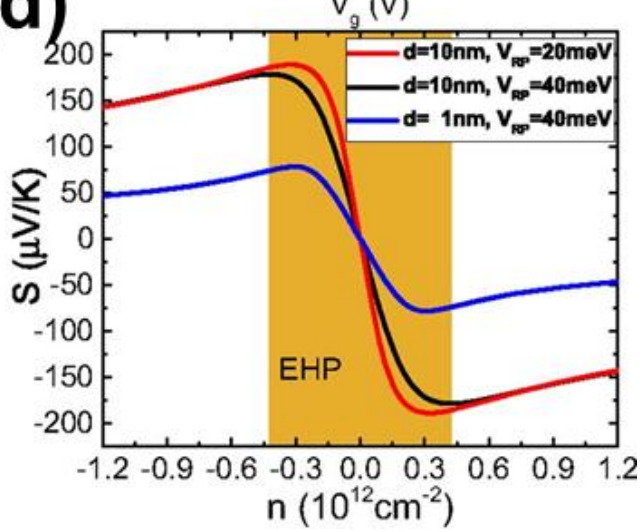

Fig. 9 A graphene/hBN heterostructure device with an enhanced in-plane $P F$. (a) Schematic of the thermoelectric measurement device. (b) Seebeck coefficients as a function of the gate voltage for $\mathrm{G} / \mathrm{hBN}$ and $\mathrm{G} / \mathrm{SiO}_{2}$ at $290 \mathrm{~K}$. (c) $P F T$ as a function of the gate voltage for $\mathrm{G} / \mathrm{hBN}$ and $\mathrm{G} / \mathrm{SiO}_{2}$ at $290 \mathrm{~K}$. (d) Simulation of the Seebeck coefficient as a function of the carrier density at $300 \mathrm{~K}$, with different hBN thicknesses, $d$, and random potential fluctuations, $V_{\mathrm{RP}}$, reproduced with permission from [5]. Copyright@The National Academy of Sciences, India. 

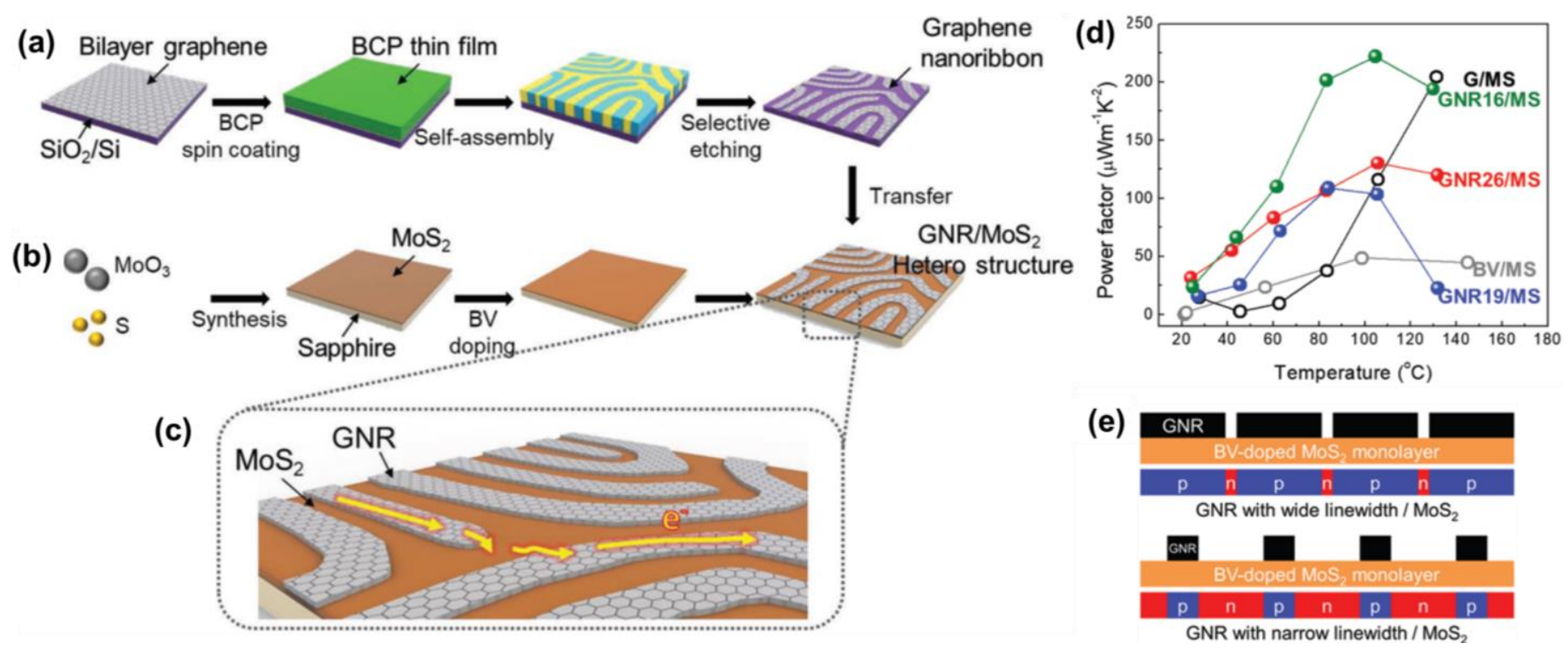

Fig. $10 \mathrm{GNR} / \mathrm{MoS}_{2}$ heterostructures with improved thermoelectric properties. (a) Fabrication of the GNR network. (b) Synthesis of doped $\mathrm{MoS}_{2}$. (c) Schematic diagram of the in-plane carrier movement across the GNR/MoS 2 van der Waals heterojunctions. (d) The $P F \mathrm{~s}$ of the GNR/MoS 2 heterostructures. (e) Schematic images of the GNR/MoS 2 heterostructures with wide and narrow GNRs. reproduced with permission from [219]. Copyright@John Wiley and Sons Ltd.

$87.7-125.7 \mu \mathrm{V} / \mathrm{K}$ at $80-220 \mathrm{~K}$, several times that of large-area graphene because of bandgap opening. As a result, the $Z T$ reached record-high values for graphene of about 0.1 , which demonstrated that nanostructured graphene can be a superior thermoelectric material for power generation as predicted in the theoretical studies.

\subsubsection{Graphene nanoconstrictions}

Harzheim and co-workers ${ }^{[261]}$ fabricated a bow-tie structure on graphene with a neck width of $100 \mathrm{~nm}$, and used a scanning thermal microscope (SThM) combined with a lock-in technique to decouple the Joule heating effect and Peltier heating/cooling effect around the nanoconstriction. The 100nm-wide nanoconstrictions in mono- and bilayer graphene exhibited a spatially correlated gradient in the Peltier coefficient and changed the Seebeck coefficient by orders of magnitude. Based on this discovery of the geometry dependent Seebeck coefficient in graphene, they further fabricated a Ushape patterned graphene consisting of one wide and one narrow leg that can act as a single-material thermocouple. ${ }^{[262]}$ The sensitivity of the U-shape graphene thermocouple increased with decreasing the leg width, and the best performance of $39 \mu \mathrm{V} / \mathrm{K}$ was measured in a structure with a narrow leg width of $200 \mathrm{~nm}$ and a short junction between the two legs.

\section{Van der Waals heterostructures for thermoelectrics}

One of the advantages of 2D materials is the ability to tune their bandgap, hence tuning the thermoelectric $P F$. The change in the bandgap could be achieved by changing the number of layers, applying strain or electric field, and changing the structural composition of the material (by hydrogenation or oxidation). For example, by reducing the number of layers of arsenic from its semimetallic bulk, one can observe a transition to a semiconducting state. The monolayer honeycomb structures of arsenic, called arsenide, is a semiconductor with an indirect bandgap of $0.831 \mathrm{eV}^{[212]}$ The electronic transport of 2D layers also depends on the type of substrate used. Via long-range Coulomb interaction, the impurities in the substrate can affect the electrons moving in the 2D layer material. The lattice constant mismatch between the substrate and the 2D layer can cause strain/stress on the 2D material and modify its electronic structure. Finally, the mismatch of the crystal structure can result in the breaking of the symmetry and can modify the electronic structure of the 2D layer. In the first part of this section, we focus on the inplane thermoelectric transport with the substrate and the number of layers as two important parameters in mind.

The discovery of 2D layered materials and the possibility of mixing and matching atomically thin layers by stacking them on top of each other, open up a new exciting opportunity. Millions of combinations of different 2D layers with different band structures are now possible to engineer the energy potential of electronic transport, enabling the design of high$P F$ structures. At the same time, due to the nature of weak van der Waals bonds, these 2D layered materials, inherently own very low thermal conductivity values in the cross-plane direction and hence pave the way for the design of solid-state thermionic structures. In the second part of this section, we focus on the cross-plane thermoelectric transport in 2D layered structures with fewer than 10 layers.

\subsection{In-plane transport}


The transport properties of 2D layers are a strong function of the substrate that they are placed on. In section 2, we focused on the thermoelectric properties of 2D layers placed on $\mathrm{SiO}_{2} / \mathrm{Si}$ substrate, which is the most widely used substrate. Here, we summarize some of the results obtained by using a different substrate or placing on $\mathrm{hBN}$ or graphene in between the $2 \mathrm{D}$ material and the $\mathrm{SiO}_{2}$ substrate.

Duan and co-workers ${ }^{[5]}$ fabricated graphene on hBN samples using the poly(methyl methacrylate) (PMMA)-based dry transfer method, and patterned metallic heater and thermometers on top of the heterostructure for the electrical conductivity and Seebeck coefficient measurements. They found that the Seebeck coefficient of graphene on $\mathrm{hBN}$ can be twice that of graphene on $\mathrm{SiO}_{2}$, and the room-temperature optimized $P F T$ value of the graphene/hBN device up to 10.35 $\mathrm{W} / \mathrm{mK}$ (Fig. 9(c)), which exceeded all the previously measured room-temperature $P F T$ values for both $3 \mathrm{D}$ and $2 \mathrm{D}$ materials. Duan et al. ${ }^{[5]}$ further demonstrated that the Seebeck coefficient offers a measure of the substrate-induced random potential fluctuations and the $\mathrm{hBN}$ substrates can enable larger bipolar switching slopes for the Seebeck coefficient (Fig. 9(b) and(d)). Combining the high $P F$ with the large thermal conductivity of both graphene and boron nitride, this work by Duan and co-workers demonstrated that the graphene/hBN heterostructure can be a very promising material for active cooling of electronics. Wu and co-workers ${ }^{[224]}$ fabricated $\mathrm{MoS}_{2} / \mathrm{hBN}$ heterostructure samples with sulfur vacancies and measured the in-plane thermoelectric properties using a similar approach as in Ref. [5]. The n-type $\mathrm{MoS}_{2}$ on hBN exhibited an extremely large positive Seebeck coefficient of 2
$\mathrm{mV} / \mathrm{K}$ in the on-state because of the magnetically induced Kondo effect. ${ }^{[24]}$ Further, by tuning the chemical potential, Wu et al. measured a $P F$ as high as $50 \mathrm{~mW} / \mathrm{m} \cdot \mathrm{K}^{2}$ at $30 \mathrm{~K}$ or PFT value of $1.5 \mathrm{~W} / \mathrm{mK}$.

By combining the nanostructuring and stacking approaches, Oh and co-workers ${ }^{[219]}$ fabricated sub-20 nm GNR arrays on monolayer $\mathrm{MoS}_{2}$. They measured the in-plane electrical conductivity and Seebeck coefficient of the GNR/MoS heterostructure using a commercial instrument and achieved a relatively high $P F$ of $222 \mu \mathrm{W} / \mathrm{mK}^{2}$. ${ }^{[219]}$

In a very interesting recent work ${ }^{[94]}$, Fig. 11 shows that the thermoelectric $P F$ of FeSe films, a high- $T_{\mathrm{c}}$ superconductor placed on $\mathrm{SrTiO}_{3}$ substrate, increases as the thickness decreases. When the films are about $1-n m$ thick, the equivalent of a bilayer, the thermoelectric $P F$ increases to values as large as $1.30 \mathrm{~W} / \mathrm{m} \cdot \mathrm{K}^{2}$ or $P F T$ value of $390 \mathrm{~W} / \mathrm{m} \cdot \mathrm{K} \cdot{ }^{[94]}$ This value is larger than anything reported before especially at such a low temperature. This large enhancement is attributed to the strong electronic correlation of $\mathrm{FeSe}$ electrons. Other possibilities such as the effect of the substrate, the ionic liquid used for gating, and the phonon drag effect are disputed by the authors but require more through theoretical insights. As mentioned in section 2, similar thickness dependence has been observed for $\mathrm{MoS}_{2}$ placed on $\mathrm{SiO}_{2}$ and $2 \mathrm{~L} \mathrm{MoS}_{2}$ possesses the best $P F^{[12,49]}$

Other than a single graphene sample, a counterflow roomtemperature $P F$ of $\sim 700 \mu \mathrm{W} / \mathrm{cm}^{\cdot} \mathrm{K}^{2}$ was reported for bilayer-graphene double layers separated by a thin $(\sim 20 \mathrm{~nm})$ boron nitride layer, where the top and bottom gate voltages are separately applied to each graphene layer. ${ }^{[233]}$
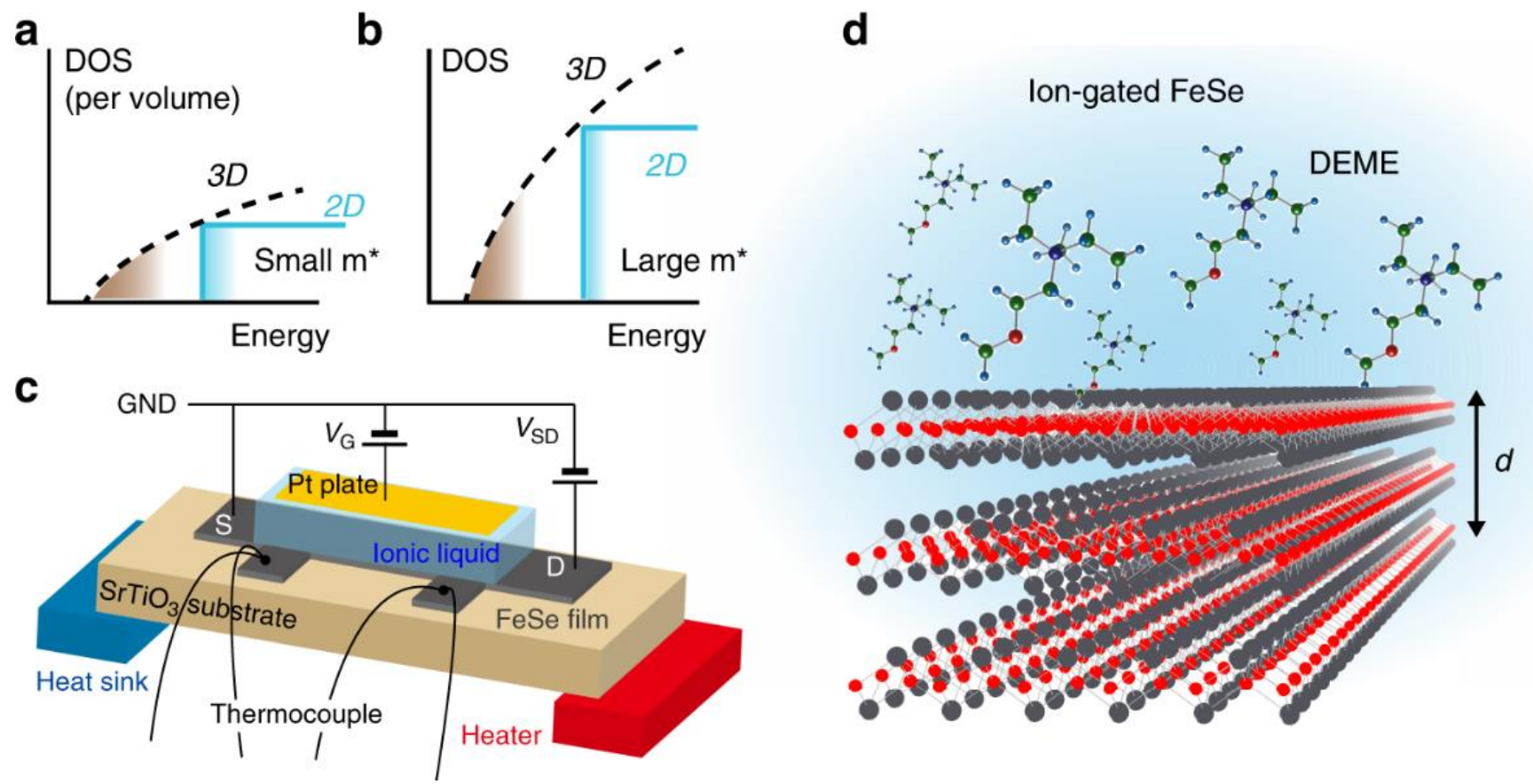

Fig. 11 Thermoelectric measurement of ion-gated FeSe thin films. (a) Schematic illustration of the DOS for 3D and 2D electrons. (b) The large effective mass $m^{*}$ enhances the DOS, which is beneficial for enhancing the Seebeck effect. (c) Device structure for thermoelectric measurement. (d) Enlarged illustration of the ionic liquid/FeSe interface, reproduced with permission from [94]. Copyright@Nature Publishing Group. 

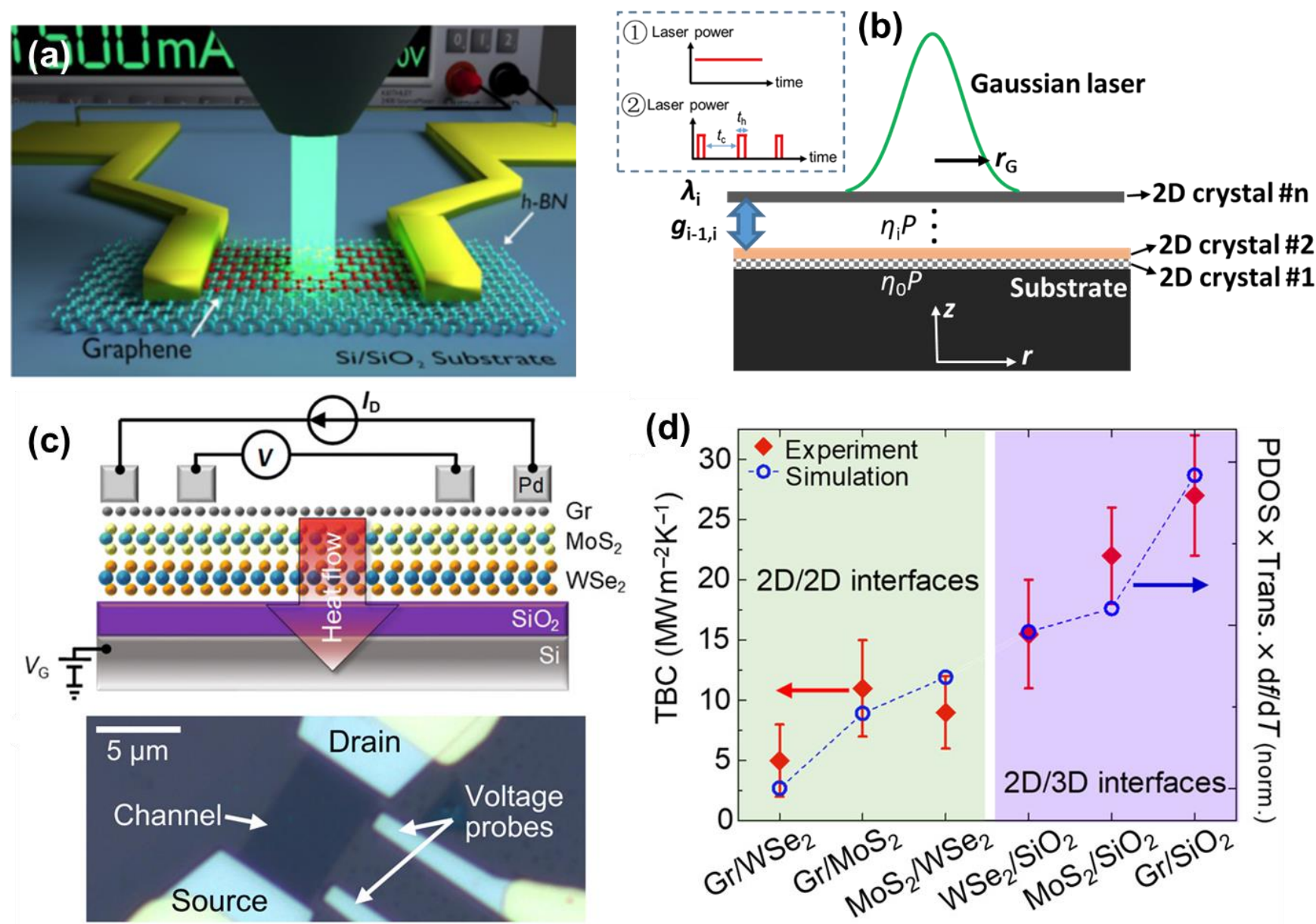

Fig. 12 Raman thermometry based thermal transport measurements of 2D van der Waals heterostructures. (a) Measurement of TBC between graphene and hBN using the Joule-heating and Raman-thermometer method, reproduced with permission from [218]. Copyright@American Institute of Physics. (b) The variable-spot-size transient Raman optothermal method to measure the in-plane and cross-plane thermal properties of van der Waals heterostructures, reproduced with permission from [213]. Copyright@Elsevier Ltd. (c) Measurement of TBC between multilayer heterostructures using the Joule-heating and Raman-thermometer method. (d) Measured room-temperature $\mathrm{TBC}$ values of 2D/2D and 2D/3D (with $\mathrm{SiO}_{2}$ ) interfaces and the calculated product of phonon $\mathrm{DOS}$, phonon transmission, and $\mathrm{d} f / \mathrm{d} T$, normalized to the minimum achieved for $\mathrm{Gr} / \mathrm{WSe}_{2}$. (c) and (d) are reproduced with permission from [214]. Copyright@American Association for the Advancement of Science.

4.2 Cross-plane transport: solid-state thermionic structure Here, we focus on 2D layered materials with metallic contacts on the top and the bottom and hence transport of electrons and phonons perpendicular to layer planes wherein an energy barrier is formed due to the band alignment of the heterostructure. When there is only a monolayer, transport is dominated by electron tunneling. Due to the transfer of low energy electrons, expected $Z T$ values of tunneling interfaces are small. The tunneling can be eliminated by increasing the number of layers. It is shown that about 4 layers are needed to suppress the tunneling effects ${ }^{[234]}$ and force the electrons to go above the energy barrier, the so-called thermionic transport is then dominant. The concept of solid-state thermionic cooling was first proposed by Shakouri ${ }^{[235]}$ and later was evaluated in both the power generation and the cooling mode by Mahan. ${ }^{[236]}$ These structures are in between vacuum state thermionic generators and thermoelectric generators. Similar to vacuum state thermionic generators, ${ }^{[237-240]}$ there is an energy barrier that electrons need to overcome to move from the hot cathode to the cold anode. The energy barrier of a vacuum state thermionic generators is determined by the work-function of the metallic cathode and anode. Since the work function of metals is on the order of $\mathrm{eV}$, temperatures larger than $1000 \mathrm{~K}$ are needed for electron transport. This problem is resolved in solid-state thermionic generators since the energy barrier can be adjusted to much lower values by proper choice of the metal-semiconductor interface. In these structures, the difference between the metal work function and the electron affinity of the semiconducting layer determines the electronic energy barrier. Hence by designing the energy barrier to few $k_{\mathrm{B}} T$, one can design the appropriate generator for the given operating temperature, $T$. In the case of 5 to 10 layers of $2 \mathrm{D}$ van der Waals heterostructure, the chemical potential is pinned by the metallic contact but the energy barrier can be 
engineered with a high degree of flexibility by finding the right sequence of layers. ${ }^{[241,242]}$

When the thickness of the $2 \mathrm{D}$ layered material is comparable or larger than the electron MFP, we see a transition from ballistic transport to diffusive transport, which is closer to thermoelectric transport. In this case, transport is mostly a function of the semiconducting layers and the effect of the contacts is minimum and limited to the electrical and thermal contact resistances at the metallic interfaces. The situation is different in solid-state thermionic generators. There, due to the ballistic nature of transport, electrons are not in near equilibrium with the lattice of the semiconducting layer. Hence, to understand thermionic transport, metallic contacts need to be included in the analysis. The combination of cathode-semiconductor-anode determines the energy barrier, the positioning of the chemical potential, and hence the thermionic $P F$.

Several theoretical works have shown the potential of 2D van der Waals heterostructures for solid-state thermionic applications and they all point to large $Z T$ values. ${ }^{[234,243-245]}$ However, all calculations are done for ideal interfaces. In practice, making such ideal clean interfaces using transfer techniques is not realistic. Hence, the future of this field should be guided by more experimental results.

In the out-of-plane direction, one of the experimental challenges is how to determine the temperature gradient across the atomic thin layers for the thermal and thermoelectric characterization. The Raman spectroscopy based non-contact thermometer enables simultaneous temperature detection of every atomic thin material from their distinct Raman peaks that shift with temperatures, ${ }^{[34,203,204,232]}$ and thus has been employed to measure the thermal boundary resistance at the van der Waals interface and the Seebeck coefficient in the cross-plane direction. Chen and co-workers ${ }^{[218]}$ stacked graphene on the insulating $\mathrm{hBN}$ and patterned electrodes on graphene to provide Joule self-heating. The Joule heat flux transferred across the heterojunction and the temperatures of both the graphene and hBN layers were simultaneously measured from the Raman spectrum (Fig. 12(a)), from which the thermal boundary conductance (TBC) between graphene and $\mathrm{hBN}$ was determined to be $7.4 \mathrm{MW} / \mathrm{m}^{2} \mathrm{~K}$. Vaziri et al. ${ }^{[214]}$ combined the Joule heating and the Raman thermometry to measure the thermal conductance of more complicated heterostructure devices involving graphene $(\mathrm{Gr}), \mathrm{MoS}_{2}$, and $\mathrm{WSe}_{2}$ (Fig. 12(c)). The TBC values at the $2 \mathrm{D} / 2 \mathrm{D}$ interfaces were measured to be $5 \pm 3 \mathrm{MW} / \mathrm{m}^{2} \mathrm{~K}, 10 \pm 3 \mathrm{MW} / \mathrm{m}^{2} \mathrm{~K}$, and $9 \pm$ $3 \mathrm{MW} / \mathrm{m}^{2} \mathrm{~K}$ for the $\mathrm{Gr} / \mathrm{WSe}_{2}, \mathrm{Gr} / \mathrm{MoS}_{2}$, and $\mathrm{MoS}_{2} / \mathrm{WSe}_{2}$ interfaces, respectively, which are significantly lower than those at the $2 \mathrm{D} / 3 \mathrm{D}\left(\mathrm{SiO}_{2}\right)$ interfaces (Fig. 12(d)). Besides, Li et al. ${ }^{[213]}$ combined laser heating and Raman thermometry, and developed a variable-spot-size transient Raman opto-thermal method to measure both in-plane and cross-plane thermal properties of 2D heterostructures in a non-contact manner (Fig $12(b))$.
Another widely studied technique for thermal boundary resistance measurement is the time domain thermo-reflectance method (TDTR). ${ }^{[246]}$ TDTR has been widely used to measure the thermal transport across various interfaces. However, only recently this method is extended to small scales enabling measurement of micron-size flakes. In the context of crossplane thermoelectric transport measurements, TDTR is recently used to measure thermal conductance across $\mathrm{Au} / \mathrm{Ti}$ (or $\mathrm{TiOx}$ )/WSe $/ \mathrm{WHOPG}_{2}$. It is shown that the TBC depends on the details of the contact, and the level of oxidization of titanium and is in the range of 6 to $22 \mathrm{MW} / \mathrm{m}^{2} \mathrm{~K}$. Despite the small thermal conductance values, the studied interface has a low $Z T$ due to the dominance of the electronic tunneling transport. ${ }^{[247]}$

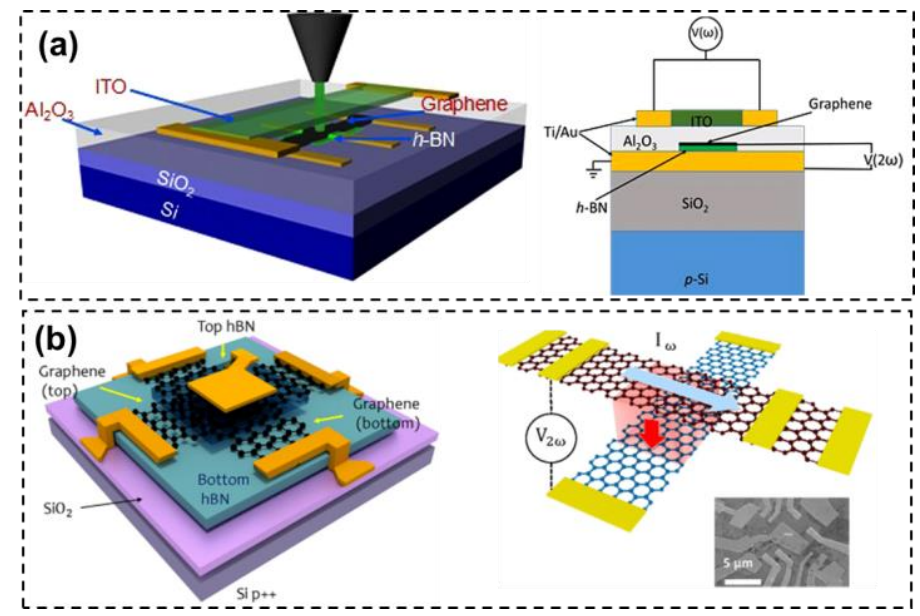
Fig. 13 Cross-plane Seebeck coefficient measurements of 2D van der Waals heterostructures. (a) Measurement of cross-plane Seebeck coefficient of graphene/hBN/Au heterostructures using Raman thermometry, reproduced with permission from [217]. Copyright@Nature Publishing Group. (b) Measurement of Seebeck coefficient across the van der Waals junction in bilayer graphene, reproduced with permission from [216]. Copyright@American Chemical Society.

A few experiments have been conducted to measure the cross-plane Seebeck coefficient of 2D heterostructures. Chen et al. ${ }^{[215]}$ stacked a $\mathrm{Gr} / \mathrm{hBN} / \mathrm{Gr}$ heterostructure and patterned electrodes on the top and bottom graphene sheets. The top graphene was fed with a sinusoidal current $(\omega)$ to provide Joule heating and establish a temperature gradient in the crossplane direction. The Seebeck voltage across the top and bottom graphene was measured from the $2 \omega$ voltage using the lock-in technique, while the temperature difference was detected by the Raman thermometry. In this way, Chen et al. comprehensively measured the thermal conductance, the Seebeck coefficient, as well as the electrical conductance in the cross-plane for the same heterostructure device. The crossplane Seebeck coefficient was $-99.3 \mu \mathrm{V} / \mathrm{K}$, the $P F\left(S^{2} G\right)$ was $1.51 \times 10^{-15} \mathrm{~W} / \mathrm{K}^{2}$, and the $Z T$ was $1.05 \times 10^{-6}$ for the $\mathrm{Gr} / \mathrm{hBN} / \mathrm{Gr}$ heterostructure. The extremely low $Z T$ is the result of the low electrical conductance, which in turn is due to the large energy barrier formed by the insulating hBN layer. Poudel and co-workers ${ }^{[217]}$ reported a modified test device 


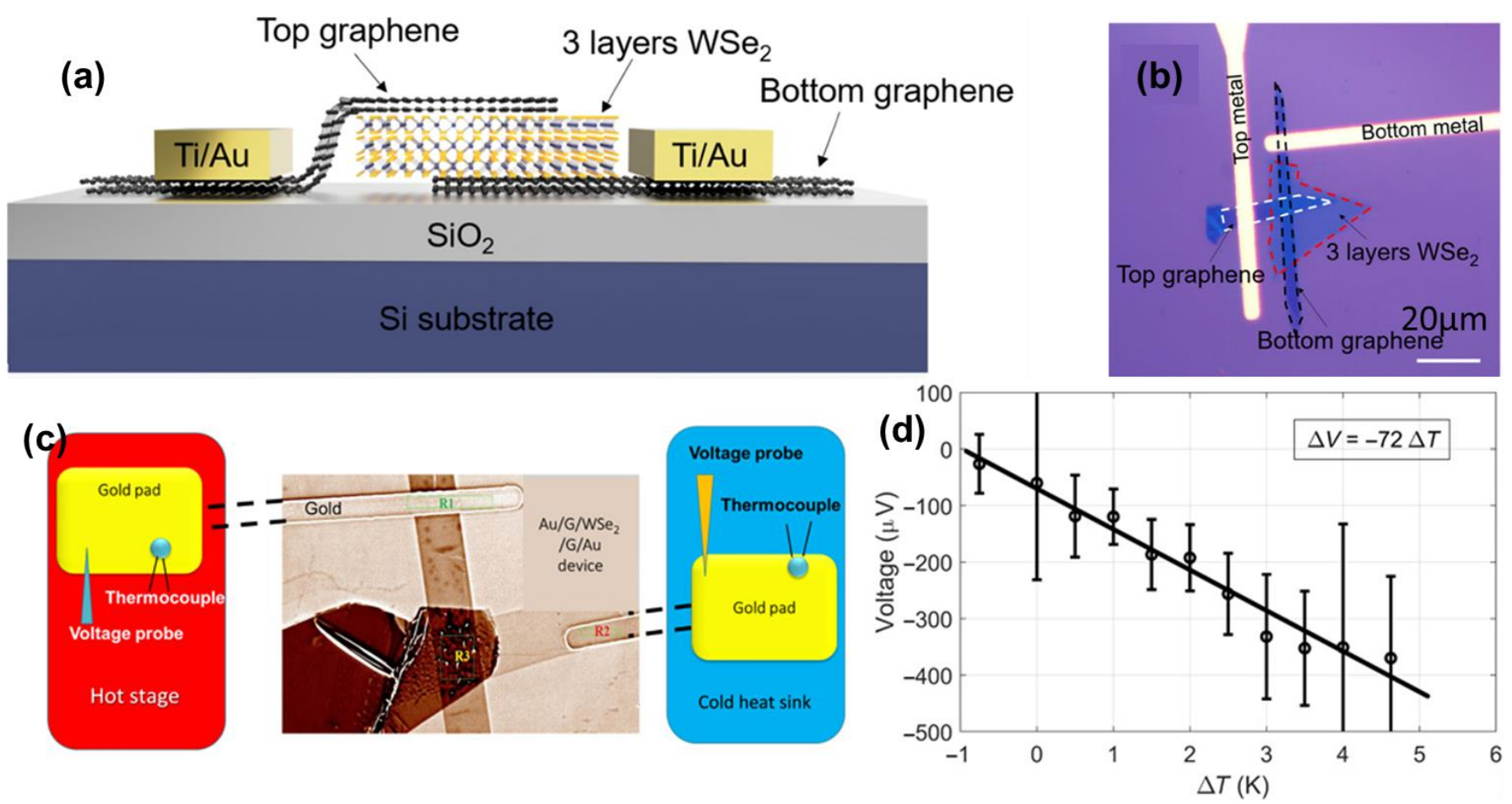

(e)

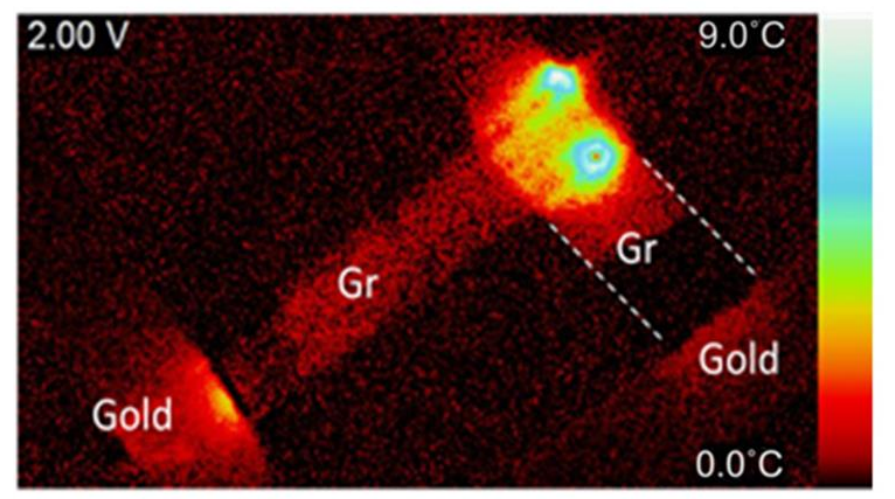

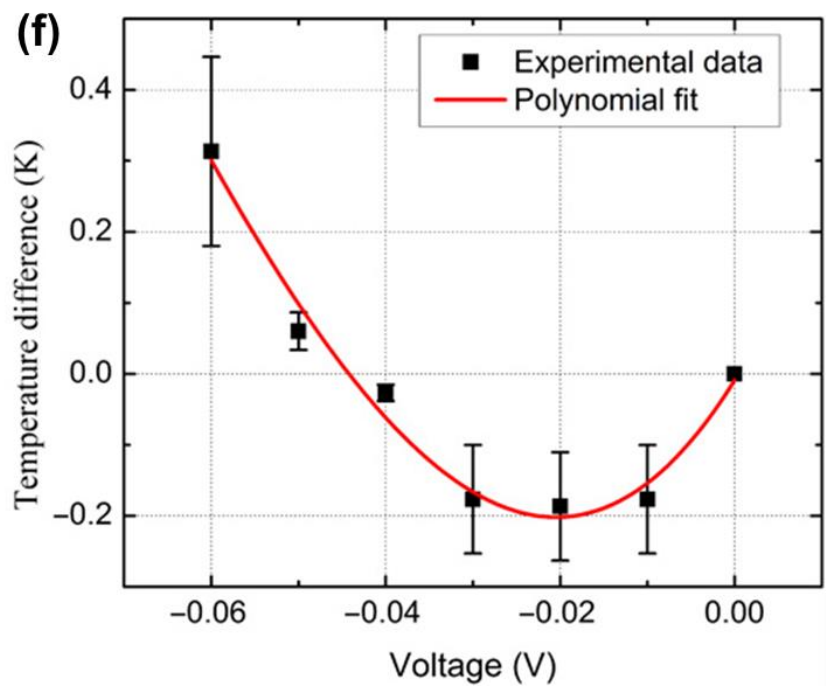

Fig. 14 Thermionic transport measurement across gold/Gr/WSe 2 van der Waals heterostructures. (a) Illustration of the cross-section of the $\mathrm{Au} / \mathrm{Gr} / \mathrm{WSe}_{2} / \mathrm{Gr} / \mathrm{Au}$ structure. (b) Optical microscope image of an $\mathrm{Au} / \mathrm{Gr} / \mathrm{WSe} / \mathrm{Gr} / \mathrm{Au}$ device. (c) Schematic of the Seebeck measurement setup. (d) Seebeck voltage measured versus applied temperature difference. (e) Joule heating: 2D temperature map of the $\mathrm{Au} / \mathrm{Gr} / \mathrm{WSe} \mathrm{S}_{2} / \mathrm{Gr} / \mathrm{Au}$ device under a relatively high-voltage $2 \mathrm{~V}$ obtained using the thermoreflectance method. (f) Cooling curve: the temperature difference between the substrate and top of the active device in Kelvin versus applied voltage at small applied voltages, reproduced with permission from [248]. Copyright@American Association for the Advancement of Science.

where the insulating $\mathrm{Al}_{2} \mathrm{O}_{3}$ and an indium tin oxide (ITO) sheets encapsulated by hBN using the layer-by-layer transparent heater were coated on the $\mathrm{Gr} / \mathrm{hBN} / \mathrm{Au}$ mechanical transfer method, as illustrated in Fig. 13(b). The heterostructure (Fig. 13(a)). Similar to the work by Chen et al., top graphene was conducted with sinusoidal current and the while the transparent top heater was driven with a sinusoidal Seebeck coefficient across the twisted bilayer graphene was current to provide heat flux in the cross-plane direction, the temperature difference between graphene and $\mathrm{hBN}$ was measured from their Raman peaks and the cross-plane Seebeck coefficient was detected from the lock-in $2 \omega$ voltage, which yieldedan interfacial Seebeck coefficient of $-215 \mu \mathrm{V} / \mathrm{K}$. Mahapatra and co-workers ${ }^{[216]}$ stacked two layers of graphene measured by the lock-in technique, while the temperatures of the two monolayers were determined from the temperature dependence of the in-plane electrical resistance of each graphene sheet. The maximum Seebeck coefficient of the van der Waals junction between graphene layers was measured to be $20-25 \mu \mathrm{V} / \mathrm{K}$ at a carrier density of $1 \times 10^{11} \mathrm{~cm}^{-2}$ at $70 \mathrm{~K}$. 
Rosul et al. ${ }^{[248]}$ used a combination of TDTR measurements for determining the thermal conductance, the thermoreflectance method for cooling curve measurements, and direct Seebeck measurement using thermocouples to study $\mathrm{Au} / \mathrm{Gr} / \mathrm{WSe}_{2} / \mathrm{Gr} / \mathrm{Au}$ structures (Fig. 14). They estimated a $Z T$ value of $10^{-3}$ at RT, which is the largest reported for nanometer-size thermionic structures. This small $Z T$ is close to the predicted $Z T$ using first-principles calculations at RT for this structure. Theoretically, it is predicted that the $Z T$ can be significantly improved by increasing the operating temperature and by changing the electrodes from gold to platinum with a lower formed energy barrier. Hence developing the transport measurement techniques enabling high-temperature measurements, and developing techniques for more flexible fabrication of a variety of different metals2D layers with clean interfaces, seem to be the most immediate need for further development of the 2D van der Waals based thermionic generators.

\section{Summary and outlook}

In parallel to the search for new materials, existing 2D materials can be tailored for their transport, optical, magnetic, and other properties simply by patterned nanostructures and stacked forms. Tremendous research opportunities exist in thermoelectric applications of 2D materials, including power generation and active cooling applications. Up to now, suspended GNRs have exhibited record-high $Z T$ values for graphene, ${ }^{[207]}$ and the device thermal resistance is larger than the on-substrate case, which is beneficial for maintaining the temperature difference. Such kind of configuration can be used for power generation as nano-generators. Nanostructured ${ }^{[196]}$ and heterostructured ${ }^{[5]}$ 2D materials supported on a substrate have exhibited enhanced $P F \mathrm{~s}$, which can be conveniently integrated into nanoelectronics for thermoelectric applications. For active cooling applications where heat is dissipated from the hot spot to the cold end using the Peltier effect, on-substrate 2D samples are better than suspended samples. In this situation, a high thermal conductivity and a high $P F$ are required, whereas $Z T$ no longer serves as the criterion for cooling performance. When a sample is placed on a substrate, heat can be dissipated into the substrate along the whole sample (i.e., effectively larger thermal conductivity due to heat leakage into the substrate), instead of heat rejection into the substrate at the junction only as in the suspended configuration.

However, there are still many important issues to be addressed. First, accurate thermoelectric measurements for such fragile samples are still challenging and caution should be taken for making the electrical/thermal contacts to the samples. For $Z T$ calculations, the same sample must be measured along the same direction for all three thermoelectric properties. This can be more difficult in some cases and an integrated measurement setup is required to simultaneously measure all properties, as demonstrated for nanoporous $\mathrm{Si}$ thin films. ${ }^{[249]}$ Second, theoretical calculations often suggested the impact of detailed atomic structures, e.g., structure disorder, chirality, zigzag or armchair edges. This has been largely neglected in measurements. A unified picture is still missing. A disconnection between theoretical studies and experimental measurements can be found wherein the theory addresses more ideal structures, and the exact nature of the defects in the experiment is not known. Similar discrepancies exist in the structure dimension, where ultrafine nanoporous 2D materials are still hard to be fabricated. In this aspect, directly grown 2D porous materials with uniform sub- $1 \mathrm{~nm}$ holes ${ }^{[250,251]}$ may allow direct comparison between theoretical and experimental studies. High-energy electron or ion beam can also be used to drill sub-1 $\mathrm{nm}$ pores but attention should be paid to the atomic structure around pores. ${ }^{[252-254]}$ Third, more advancements are required in first-principles predictions of the transport properties. For the materials genome, a high-throughput calculation method is required and this can be achieved with machine learning. Successful examples for machine learning in materials search and design can be found for interfacial thermal conductance, ${ }^{[175,255,256]}$ bandgap, ${ }^{[257]}$ and interatomic force constants ${ }^{[258,259,260]}$ used in MD simulations. Machine learning driven by experimental data is desired for thermoelectric studies but is still lacking due to the challenge of high-throughput measurements at the nanoscale. Finally, the device-level applications of these materials and their mass production should receive more attention. The high nanofabrication cost of some nanostructured or stacked 2D materials may hinder their wide applications.

\section{Acknowledgments}

This work was partially supported by JSPS KAKENHI (Grant No. JP20H02090) and the JST CREST Grant Number JPMJCR18I1, Japan. Hao acknowledges the support from NSF (grant number CBET-1803931). Zebarjadi and Zhu acknowledge the support from NSF grant number 1653268.

\section{Supporting Information}

Not applicable

\section{Conflict of Interest}

There are no conflicts to declare.

\section{References}

[1] P. Dollfus, V. H. Nguyen and J. Saint-Martin, J Phys. Condens. Matter, 2015, 27, 133204, doi:10.1088/0953-8984/27/13/133204.

[2] M. Markov and M. Zebarjadi, Nanosc. Microsc. Therm. Eng., 2019, 23, 117-127, doi: 10.1080/15567265.2018.1520762.

[3] M. Zebarjadi, Electronic cooling using thermoelectric devices, Appl. Phys. Lett., 2015, 106, 203506, doi: 10.1063/1.4921457.

[4] M.J. Adams, M. Verosky, M. Zebarjadi and J.P. Heremans, Phys. Rev. Appl., 2019, 11, 054008, doi: 10.1103/PhysRevApplied.11.054008.

[5] J. Duan, X. Wang, X. Lai, G. Li, K. Watanabe, T. Taniguchi, M. Zebarjadi and E.Y. Andrei, Proc. Natl. Acad. Sci., 2016, 113, 14272-14276, doi: 10.1073/pnas.1615913113. 
[6] M. S. Lundstrom and C. Jeong, Near-Equilibrium Transport: Fundamentals and Applications 2012, 2, World Scientific Publishing Company.

[7] C. Jeong, R. Kim, M. Luisier, S. Datta and M. Lundstrom, J Appl.Phys., 2010, 107, 023707, doi: 10.1063/1.3291120.

[8] Fisher and S. Timothy Thermal energy at the nanoscale. 2013, 3, World Scientific Publishing Company.

[9] G. D. Mahan and J. O. Sofo, The best thermoelectric. Proc. Natl. Acad. Sci., 1996, 93, 7436-7439.

[10] J. H. Seol, I. Jo, A. L. Moore, L. Lindsay, Z. H. Aitken, M. T. Pettes, X. Li, Z. Yao, R. Huang, D. Broido, N. Mingo, R. S. Ruoff, L. Shi, Science, 2010, 328, 213-216, doi: 10.1126/science. 1184014 .

[11] Y. M. Zuev, W. Chang and P. Kim, Phys. Rev. Lett., 2009, 102, 096807, doi: 10.1103/PhysRevLett.102.096807.

[12] K. Hippalgaonkar, Y. Wang, Y. Ye, D. Y. Qiu, H. Zhu, Y. Wang, J. Moore, S. G. Louie and X. Zhang, Phys. Rev. B, 2017, 95, 115407, doi: 10.1103/PhysRevB.95.115407.

[13] L. D. Hicks, and M. S. Dresselhaus, Phys. Rev. B, 1993, 47 12727, doi: 10.1103/PhysRevB.47.12727.

[14] L. D. Hicks, and M. S. Dresselhaus, Phys. Rev. B, 1993, 47, 16631, doi: 10.1103/PhysRevB.47.16631.

[15] J. Mao, Z. Liu and Z. Ren, NPJ Quantum Material., 2016, 1, 1-9, doi: 10.1038/npjquantmats.2016.28.

[16] Y. Xiao, Q. Chen, D. Ma, N. Yang and Q. Hao, ES Mater. Manuf., 2019, 5, 2-18, doi: 10.30919/esmm5f237.

[17] K. S. Novoselov, A. Mishchenko, A. Carvalho and A. C. Neto, Science, 2016, 353, 6298, aac9439, doi: 10.1126/science.aac9439.

[18] A. K. Geim and I. V. Grigorieva, Nature, 2013, 499, 419-425, doi: 10.1038/nature12385.

[19] N. Mounet, M. Gibertini, P. Schwaller, D. Campi, A. Merkys, A. Marrazzo, T. Sohier, I. E. Castelli, A. Cepellotti, G. Pizzi and N. Marzari, Nat. Nanotechnol., 2018, 13, 246-252, doi: 10.1038/s41565-017-0035-5.

[20] Y. Xu, Z. Li, and W. Duan. Small, 2014, 10, 2182-2199, doi: 10.1002/smll.201303701.

[21] G. Zhang and Y. W. Zhang, J. Mater. Chem. C, 2017, 5, 7684-7698, doi: 10.1039/C7TC01088E.

[22] J. Wu, Y. Chen, J. Wu, K. Hippalgaonkar. Adv. Electron. Mater., 2018, 4, 1800248, doi: 10.1002/aelm.201800248.

[23] Y. Zhao, Y. Cai, L. Zhang, B. Li, G. Zhang, J. T. Thong, Adv. Funct. Mater, $2020, \quad$ 30, 8 1903929, doi: 10.1002/adfm.201903929.

[24] K. Kanahashi, J. Pu, Jiang, T. Takenobu, Adv. Energy Mater., 2020, 10, 1902842, doi: 10.1002/aenm.201902842.

[25] P. A. Zong, J. Liang, P. Zhang, C. Wan, Y. Wang and K. Koumoto, ACS Appl. Energy Mater., 2020, 3, 2224-2239, doi: 10.1021/acsaem.9b02187.

[26] D. Li, Y. Gong, Y. Chen, J. Lin, Q. Khan, Y. Zhang, Y. Li, H. Zhang and H. Xie. Recent Progress of Two-Dimensional Thermoelectric Materials. Nano-Micro Lett., 2020, 12, 36, doi: 10.1007/s40820-020-0374-x.

[27] N. S. Sankeshwar, S. S. Kubakaddi and B. G. Mulimani, Advances in Graphene Science, 2013, 10, 56720, doi:
$10.5772 / 56720$

[28] P. Wei, W. Bao, Y. Pu, C. Lau and J. Shi, Phys. Rev. Lett., 2009, 102, 166808, doi: 10.1103/PhysRevLett.102.166808.

[29] J. Checkelsky and N. Ong, Phys. Rev. B, 2009, 80, 081413, doi: 10.1103/PhysRevB.80.081413.

[30] X. Wu, Y. Hu, M. Ruan, N K Madiomanana, C Berger and W. A. de Heer, Appl. Phys. Lett., 2011, 99, 133102.

[31] M. M. Sadeghi, I. Jo and L. Shi, Proc. Natl. Acad. Sci., 2013, 110, 16321-16326.

[32] E. Pop, V. Varshney and A. K. Roy, MRS Bull., 2012, 37(12), 1273-1281, doi: 10.1557/mrs.2012.203

[33] A. A. Balandin, Nat. Mater, 2011, 10, 569-581, doi: https://doi.org/10.1038/nmat3064

[34] Q. Y. Li, K. Xia, J. Zhang, Y. Zhang, Q. Li, K. Takahashi and X. Zhang, Nanoscale, 2017, 9, 10784-10793, doi: 10.1039/C7NR01695F.

[35] X.Gu, Z. Fan, H. Bao and C. Y. Zhao, Phys. Rev. B, 2019, 100, 064306, doi: 10.1103/PhysRevB.100.064306.

[36] Y. Zhang, T. T. Tang, C. Girit, Z. Hao, M. C. Martin, A. Zettl, M.F. Crommie, Y. Ron Shen and F. Wang, Nature, 2009, 459, 820-823, doi: 10.1038/nature08105.

[37] C. H. Lui, Z. Li, K. F. Mak, E. Cappelluti and T. F. Heinz, Nat. Phys., 2011, 7, 944-947, doi: 10.1038/nphys 2102.

[38] W. Bao, L. Jing, J. Velasco, Y. Lee, G. Liu, D. Tran, B. Standly, M. Aykol, S. B. Cronin, D. Smirnov, M. Koshino, E. McCann, M. Bockrath and C. N. Lau, Nat. Phys., 2011, 7, 948952, doi: 10.1038/nphys2103.

[39] Y. Cao, V. Fatemi, S. Fang, K. Watanabe, T. Taniguchi, E. Kaxiras and P. Jarillo-Herrero, Nature, 2018, 556, 43-50, doi: 10.1038/nature26160

[40] S-G Nam, D-K Ki and H-J Lee, Phys. Rev. B, 2010, 82, 245416, doi: 10.1103/PhysRevB.82.245416.

[41] C. R. Wang, W. S. Lu, L. Hao, W. L. Lee, T. K. Lee, F. Lin, I. C. Cheng and J. Z. Chen, Enhanced thermoelectric power in dual-gated bilayer graphene. Phys. Rev. Lett., 2011 107, 186602, doi: 10.1103/PhysRevLett.107.186602.

[42] Y. Y. Chien, H. Yuan, C. R. Wang, and W. L. Lee, Sci. Rep., 2016, 6, 20402, doi: 10.1038/srep20402.

[43] D. Suszalski, G. Rut, and A. Rycerz, J Phys. Condens. Matter., 2019, 31, 415501, doi: 10.1088/1361-648X\%2Fab2d0c. [44] S. Manzeli, D. Ovchinnikov, D. Pasquier, O. V. Yazyev andA.Kis, Nat. Rev. Mater, 2017, 2, 17033, doi: 10.1038/natrevmats.2017.33.

[45] W. Huang, H. Da and Liang, G., J. Appl. Phys., 2013, 113, 104304, doi: 10.1063/1.4794363.

[46] D. Wickramaratne, F. Zahid, R. K. Lake, J. Chem. Phys., 2014, 140, 124710, doi: 10.1063/1.4869142.

[47] W. Huang, X. Luo, C. K. Gan, S. Y. Quek, S. Y. and G. Liang, Phys. Chem. Chem. Phys., 2014, 16, 10866-10874, doi: 10.1039/C4CP00487F.

[48] J. Wu, H. Schmidt, K. K. Amara, X. Xu, G. Eda and B. Özyilmaz, Nano Lett., 2014, 145, 2730-2734, doi: $10.1021 / \mathrm{nl} 500666 \mathrm{~m}$

[49] M. Kayyalha, J. Maassen, M. Lundstrom, L. Shi, Y.P. Chen, J. Appl. Phys., 2016, 120, 134305, doi: 10.1063/1.4963364. 
[50] Z. Jin, Q. Liao, H. Fang, Z. Liu, W. Liu, Z. Ding, T. Luo and N. Yang, Sci. Rep., 2015, 5, 18342, doi: 10.1038/srep18342.

[51] Z. Li, Y. Lv, L. Ren, J. Li, L. Kong, Y. Zeng, Q. Tao, R. Wu, H. Ma, B. Zhao, D. Wang, K. Chen, L. Liao, X. Duan, X. Duan and D. Wang, Nat. Commun., 2020, 11, 1-8, doi: 10.1038/s41467020-15023-3.

[52] M. Yoshida, T. Iizuka, Y. Saito, M. Onga, R. Suzuki, Y. Zhang, Y. Iwasa and S. Shimizu, Nano Lett., 2016, 16, 2061-2065, doi: 10.1021/acs.nanolett.6b00075.

[53] J. Liu, G. M. Choi and D. G. Cahill, J. Appl. Phys., 2014, 116, 233107, doi: 10.1063/1.4904513.

[54] P. Jiang, X. Qian, X. Gu and R. Yang, Adv. Mater., 2017, 29, 1701068, doi: 10.1002/adma.201701068.

[55] C. Chiritescu, D. G. Cahill, N. Nguyen, D. Johnson, A. Bodapati, P. Keblinski, P. Zschack, Science, 2007, 315, 351-353, doi: 10.1126/science.1136494.

[56] D. O. Lindroth and P. Erhart, Phys. Rev. B, 2016, 94, 115205, doi: 10.1103/PhysRevB.94.115205.

[57] R. Yan, J. R. Simpson, S. Bertolazzi, J. Brivio, M. Watson, X. Wu, A. Kis, T. Luo, A. R. Hight Walker and H. G. Xing, ACS Nano, 2014, 8, 986-993, doi: 10.1021/nn405826k.

[58] Y. Yu, T. Minhaj, L. Huang, Y. Yu, L. Cao, Phys. Rev. Appl., 2020, 13, 034059, doi: 10.1103/PhysRevApplied.13.034059.

[59] P. Yuan, R. Wang, T. Wang, X. Wang, and Y. Xie, Phys. Chem. Chem. Phys., 2018, 20, 25752-25761, doi: 10.1039/C8CP02858C.

[60] P. Yuan, R. Wang, H. Tan, T. Wang and X. Wang, ACS Photonics, 2017, 4, 3115-3129, doi: 10.1021/acsphotonics.7b00815.

[61] R. Wang, T. Wang, H. Zobeiri, P. Yuan, C. Deng, Y. Yue, S. $\mathrm{Xu}$ and $\mathrm{X}$. Wang, Nanoscale, 2018, 10, 23087-23102, doi: 10.1039/C8NR05641B.

[62] R. Wang, H. Zobeiri, Y. Xie, X. Wang, X. Zhang and Y. Yue, Adv. Sci., 2020, 2000097, doi: 10.1002/advs.202000097.

[63] N. Peimyoo, J. Shang, W. Yang, Y. Wang, C. Cong, and T. Yu, NanoRes., 2015, 8, 1210, doi: 10.1007/s12274-014-0602-0. [64] I. Jo, M. T. Pettes, E. Ou, W. Wu and L. Shi, Appl. Phys. Lett., 2014, 104, 201902, doi: 10.1063/1.4876965.

[65] M. Yarali, X. Wu, T. Gupta, D. Ghoshal, L. Xie, Z. Zhu, H. Brahmi, J. Bao, S. Chen, T. Luo and N. Koratkar, Adv. Funct. Mater, 2017, 27, 1704357, doi: 10.1002/adfm.201704357.

[66] A. Aiyiti, X. Bai, J. Wu, X. Xu and B. Li, Sci. Bull. 2018, 637, 452-458, doi: 10.1016/j.scib.2018.02.022.

[67] X.Gu and R. Yang, Appl. Phys. Lett., 2014, 105, 131903, doi: $10.1063 / 1.4896685$.

[68] K. Xu, A. J. Gabourie, A. Hashemi, Z. Fan, N. Wei, A. B. Farimani, H. P. Komsa, A.V. Krasheninnikov, E. Pop, and T. Ala-Nissila, Phys. Rev. B, 2019, 99, 054303, doi: 10.1103/PhysRevB.99.054303.

[69] X. Gu, B. Li and R. Yang, J. Appl. Phys., 2016, 119, 085106, doi: $10.1063 / 1.4942827$.

[70] W. X. Zhou and and K. Q. Chen, Sci. Rep., 2015, 5, 15070, doi: $10.1038 /$ srep 15070 .

[71] C. Wan, X. Gu, F. Dang, T. Itoh, Y. Wang, H. Sasaki, M. Kondo, K. Koga, K. Yabuki, G. J. Snyder, and R. Yang, Nat.
Mater., 2015, 14, 622-627, doi: 10.1038/nmat4251.

[72] L. Li, Y. Yu, G. J. Ye, Q. Ge, X. Ou, H. Wu, D. Feng, X. H. Chen, and Y. Zhang, Nat. Nanotechnol., 2014, 9, 372, doi: 10.1038/nnano.2014.35

[73] Z. Luo, J. Maassen, Y. Deng, Y. Du, R. P. Garrelts, M. Lundstrom, D. Y. Peide and X. Xu, Nat. Commun., 2015, 6,1-8, doi: $10.1038 /$ ncomms9572.

[74] H. Jang, J.D. Wood, C.R. Ryder, M. C. Hersam and D. G. Cahill, Adv. Mater, 2015, 27, 8017-8022, doi: 10.1002/adma.201503466.

[75] S. Lee, F. Yang, J. Suh, S. Yang, Y. Lee, G. Li, H. S. Choe, A. Suslu, Y. Chen, C. Ko, J. Park, K. Liu, J. Li, K. Hippalgaonkar, J. J. Urban, S. Tongay, J. Wu, Nat. Commun., 2015, 6, 1-7, doi: 10.1038/ncomms9573.

[76] B. Smith, B. Vermeersch, J. Carrete, E. Ou, J. Kim, N. Mingo, D. Akinwande and L. Shi, Adv. Mater, 2017, 29, 1603756, doi: 10.1002/adma.201603756.

[77] H. Y. Lv, W. J. Lu, D. F. Shao and Y. P. Sun, Phys. Rev. B, 2014, 90, 085433, doi: 10.1103/PhysRevB.90.085433.

[78] G. Qin, Q. B. Yan, Z. Qin, S. Y. Yue, H. J. Cui, Q. R. Zheng and G. Su, Sci. Rep., 2014, 4, 6946, doi: 10.1038/srep06946.

[79] E. Flores, J. R. Ares, A. Castellanos-Gomez, M. Barawi, I. J. Ferrer and C. Sánchez, Appl. Phys. Lett., 2015, 106, 022102, doi: 10.1063/1.4905636.

[80] Y. Saito, T. Iizuka, T. Koretsune, R. Arita, S. Shimizu, Y. Iwasa, Nano Lett., 2016, 16, 4819-4824, doi: 10.1021/acs.nanolett.6b00999.

[81] R. Fei, A. Faghaninia, R. Soklaski, J. A. Yan, C. Lo and L. Yang, Nano Lett., 2014, 14, 6393-6399, doi: 10.1021/n1502865s. [82] S.J. Choi, B.K. Kim, T. H. Lee, Y. H. Kim, Z. Li, E. Pop, J. J. Kim, J. H. Song and M. H. Bae, Nano Lett., 2016, 16, 39693975, doi: 10.1021/acs.nanolett.5b04957.

[83] M. Zare, B. Z. Rameshti, F. G. Ghamsari and R. Asgari, Phys Rev. B, 2017, 95, 045422, doi: 10.1103/PhysRevB.95.045422.

[84] L.D. Zhao, S.H. Lo, Y. Zhang, H. Sun, G. Tan, C. Uher, C. Wolverton, V. P. Dravid and M. G. Kanatzidis, Nature,2014, 508, 373-377, doi: 10.1038/nature13184.

[85] S. H. Heo, S. Jo, H. S. Kim, G. Choi, J. Y. Song, J. Y. Kang, N. J. Park, H. W. Ban, F. Kim, H. Jeong and J. Jung, Nat. Commun., 2019, 10, 1-10, doi: 10.1038/s41467-019-08883-x.

[86] X. L. Shi, X. Tao, J. Zou and Z. G. Chen, Adv. Sci., 2020, 7, 1902923, doi: 10.1002/advs.201902923.

[87] L. D. Zhao, C. Chang, G. Tan and M. G. Kanatzidis, Energy Environ. Sci., 2016, 9, 3044-3060, doi: 10.1039/C6EE01755J.

[88] Y. Zhou and L. D. Zhao, Adv. Mater., 2017, 29, 1702676, doi: 10.1002/adma.201702676.

[89] S. Lin, W. Li, Z. Chen, J. Shen, B. Ge and Y. Pei, Nat. Commun., 2016, 7, 1-6, doi: 10.1038/ncomms10287.

[90] S. Sharma, N. Singh and U. Schwingenschlögl, ACS Appl. Energy Mater. 2018, 1, 1950-1954, doi: 10.1021/acsaem.8b00032.

[91] Z. Gao, G. Liu and J. Ren, ACS Appl. Mater. Interfaces, 2018 , 10, 40702-40709, doi: 10.1021/acsami.8b11836.

[92] G. Qiu, S. Huang, M. Segovia, P. K. Venuthurumilli, Y. Wang, W. Wu, X. Xu, and P. D. Ye, Nano Lett., 2019, 19, 1955 - 
1962, doi: 10.1021/acs.nanolett.8b05144.

[93] X. Zhang, C. Liu, Y. Tao, Y. Li, Y. Guo, Y. Chen, X. C. Zeng and J. Wang, Adv. Funct. Mater.,2020, 30, 2001200, doi: 10.1002/adfm.202001200.

[94] S. Shimizu, J. Shiogai, N. Takemori, S. Sakai, H. Ikeda, R. Arita, T. Nojima, A. Tsukazaki, Y. Iwasa, Nat.Commun., 2019, 10, doi: 10.1038/s41467-019-08784-z.

[95] M.J. Lee, J.H. Ahn, J.H. Sung, H. Heo, S. G. Jeon, W. Lee, J.Y. Song, K.H. Hong, B. Choi, S.H. Lee, and M. H. Jo, Nat. Commun., 2016, 7, 1-7, doi: 10.1038/ncomms12011.

[96] S. Shimizu, M. S. Bahramy, T. Iizuka, S. Ono, K. Miwa, K., Y. Tokura and Y. Iwasa, Proc. Natl. Acad. Sci., 2016, 113, $6438-$ 6443, doi: 10.1073/pnas.1525500113.

[97] N. T. Hung, A. R. Nugraha and R. Saito, Appl. Phys. Lett., 2017, 111, 092107, doi:10.1063/1.5001184

[98] S. Sharma, S. Kumar, and U. Schwingenschlögl, Phys. Rev. Appl., 2017, 8, 044013, doi: 10.1103/PhysRevApplied.8.044013. [99] J. Yu, T. Li and Q. Sun, J. Appl. Phys., 2019, 125, 205111, doi: 10.1063/1.5098826.

[100] K. Esfarjani, G. Chenand H. T. Stokes, Phys. Rev. B, 2011, 84, 085204, doi: 10.1103/PhysRevB.84.085204.

[101] W. Zhang, T. Fisher and N. Mingo, Numer. Heat Trans. Part B, 2007, 51, 333-349, doi: 10.1080/10407790601144755.

[102] S. Datta, Electronic Transport in Mesoscopic Systems (Cambridge University Press, 1997).

[103] A. Cepellotti, G. Fugallo, L. Paulatto, M. Lazzeri, F. Mauri, N. Marzari, Nat. Commun., 2015, 6, 1-7, doi: 10.1038/ncomms7400.

[104] S. Lee, D. Broido, K. Esfarjani, G. Chen, Nat. Commun., 2015, 6, 1-10, doi: 10.1038/ncomms7290.

[105] S. Lee and X. Li (invited book chapter) Hydrodynamic Phonon Transport: Past, Present, and Prospect. In Nanoscale Energy Transport: Emerging Phenomena, Methods, and Applications, Liao, B. (Ed.). Institute of Physics Publishing, 2019 [106] X. Li and S. Lee, Phys. Rev. B, 2019, 99, 085202, doi: 10.1103/PhysRevB.99.085202.

[107] L. P. Mezhov-Deglin, J. Exp. Theor. Phys., 1965, 49, 6679.

[108] S. Huberman, R. A. Duncan, K. Chen, B. Song, V. Chiloyan, Z. Ding, A. A. Maznev, G. Chen and K. A Nelson, Science, 2019, 364, 375-379, doi: 10.1126/science.aav3548.

[109] M. Y. Shang, C. Zhang, Z. Guo, and J. T. Lü, Sci. Rep., 2020, 10, 1-10, doi: 10.1038/s41598-020-65221-8.

[110] X. M. Wang, D. C. Mo and S. S. Lu, J. Chem. Phys., 2013, 138, 204704, doi: 10.1063/1.4806069.

[111] X.Gu and R. Yang, Annu. Rev. Heat Trans., 2016, 19, 1-65, doi: 10.1615/AnnualRevHeatTransfer.2016015491.

[112] W.J. Evans, L. Hu, and P. Keblinski, Appl. Phys. Lett., 2010 96, 203112, doi: 10.1063/1.3435465.

[113] T. Feng, X. Ruan, Z. Ye, Z. and B Cao, Phys. Rev. B, 2015,91,224301, doi: 10.1103/PhysRevB.91.224301.

[114] Ç. Ö. Girit, J. C. Meyer, R. Erni, M. D. Rossell, C. Kisielowski, L. Yang, C.-H. Park, M. Crommie, M. L. Cohen and S. G. Louie, Science, 2009, 323, 1705-1708, doi: 10.1126/science. 1166999 .
[115] Z. Guo, D. Zhang, and X.-G. Gong, Appl. Phys. Lett., 2009, 95,163103, doi: 10.1063/1.3246155.

[116] E. Munoz, J. Lu, J. and B. I. Yakobson, Nano Lett., 2010, 10, 1652-1656, doi:10.1021/n1904206d.

[117] Z. Wei, Y. Chen, and C. Dames, J. Appl. Phys., 2012, 112, 024328, doi: 10.1063/1.4740065.

[118] Y. Ouyang and J. Guo, Appl. Phys. Lett., 2009, 94, 263107, doi: 10.1063/1.3171933.

[119] H. Sevinçli and G. Cuniberti, Phys. Rev. B, 2010, 81, 113401, doi: 10.1103/PhysRevB.81.113401.

[120] J. Haskins, A. Kınacı, C. Sevik, H. Sevinçli, G. Cuniberti and T. Çağın, ACS Nano, 2011, 5, 3779-3787, doi:10.1021/nn200114p.

[121] H. Karamitaheri, N. Neophytou, M. Pourfath, R. Faez, and H. Kosina, J. Appl. Phys., 2012, 111, 05450, doi: $10.1063 / 1.3688034$.

[122] H. Zheng, H. J. Liu, X. J. Tan, H. Y. Lv, L. Pan, J. Shi, and X. F. Tang, Appl. Phys. Lett., 2012, 100, 093104, doi: 10.1063/1.3689780.

[123] V. T. Tran, J. Saint-Martin, P. Dollfus, S. Volz, Sci. Rep., 2017, 7, 1-11, doi: 10.1038/s41598-017-02230-0.

[124] S. Tan, K. Q. Chen, Carbon, 2015, 94, 942-945, doi: 10.1016/j.carbon.2015.07.083.

[125] H. Sevinçli, C. Sevik, T. Çağın and G. Cuniberti, G. Sci. Rep., 2013, 3, 1-6, doi: 10.1038/srep0122.

[126] C. N. Pan, Z. X. Xie, L. M. Tang and K. Q. Chen, Appl. Phys. Lett., 2012, 101, 103115, doi: 10.1063/1.4751287.

[127] F. Mazzamuto, V. H. Nguyen, Y. Apertet, C. Caër, C. Chassat, J. Saint-Martin and P. Dollfus, Phys. Rev. B, 2011, 8323, 235426, doi: 10.1103/PhysRevB.83.235426.

[128] P. S. E. Yeo, M. B. Sullivan, K. P. Loh and C. K. Gan, J. Mater. Chem. A, 2013, 1, 10762-10767, doi: 10.1039/C3TA12211E.

[129] N. Wei, L. Xu, H. Q. Wang and J. C. Zheng, Nanotechnology, 2011, 22, 105705, doi: 10.1088/09574484/22/10/105705/meta.

[130] L. Liang, E. Cruz-Silva, E. C. Girão, V Meunier, Phys. Rev.B, 2012, 86, 115438, doi: 10.1103/PhysRevB.86.115438.

[131] F. Mazzamuto, J. Saint-Martin, V. H. Nguyen, V. H., C. Chassat, P. Dollfus, J. Comput. Electron., 2012, 11, 67-77, doi: 10.1007/s10825-012-0392-0.

[132] Y. Yan, Q. F. Liang, H. Zhao and C. Q. Wu, Phys. Lett. A, 2012, 376, 1154-1158, doi: 10.1016/j.physleta.2012.02.013.

[133] D. Ma, X. Wan and N. Yang, N. Phys. Rev. B, 2018, 98, 245420, doi: 10.1103/PhysRevB.98.245420.

[134] D. L. Nika, A. I. Cocemasov, C. L. Isacova, A. A. Balandin, V. M. Fomin and O. G. Schmidt, Phys. Rev. B, 2012, 85, 205439 , doi: 10.1103/PhysRevB.85.205439.

[135] B. L. Davis and M. I. Hussein, Phys. Rev. Lett., 2014, 112, 055505, doi: 10.1103/PhysRevLett.112.055505.

[136] X. Ni, G. Liang, J. S. Wang and B. Li, Appl. Phys. Lett., 2009, 95, 192114, doi: 10.1063/1.3264087.

[137] K. Yang, S. Cahangirov, A. Cantarero, A. Rubio and R. D'Agosta, Phys. Rev. B, 2014, 89, 125403, doi: 10.1103/PhysRevB.89.125403. 
[138] K. Zberecki, M. Wierzbicki, J. Barnaś and R. Swirkowicz, Phys. Rev. B, 2013, 88, 115404, doi: 10.1103/PhysRevB.88.115404.

[139] K. Zberecki, R. Swirkowicz and J. Barnaś, Phys. Rev. B, 2014, 89,165419, doi: 10.1103/PhysRevB.89.16541.

[140] D. D. Fan, H. J. Liu, L. Cheng, P. H. Jiang, J. Shi and X. F. Tang, Appl. Phys. Lett., 2014, 105, 133113, doi: 10.1063/1.4897349.

[141] K. X. Chen, Z. Y. Luo, D. C. Mo, and S. S. Lyu, Phys. Chem. Chem. Phys., 2016, 18, 16337-16344, doi: 10.1039/C6CP02456D. [142] J. Wang, F. Xie, X. Cao, S. C. An, W. Zhou, L. M. Tang and K. Q. Chen, Sci. Rep., 2017, 7, 1-8, doi: 10.30919/es8d1136.

[143] J. Zhang, H. J. Liu, L. Cheng, J. Wei, J. H. Liang, D. D. Fan, J. Shi, X. F. Tang, and Q. J. Zhang, Sci. Rep., 2014, 4, 1-8, doi: $10.1038 /$ srep06452.

[144] X. K. Chen, Z. X. Xie, W. X. Zhou, L. M. Tang, K. Q. Chen, Appl. Phys. Lett., 2016, 109, 023101, doi: 10.1063/1.4958688.

[145] I. M. Felix and L. F. C. Pereira, Sci. Rep., 2018, 8, 2737, doi: 10.1038/s41598-018-20997-8.

[146] V. T. Tran, J. Saint-Martin, and P. Dollfus, Nanotechnology, 2015, 26, 495202, doi: 10.1088/0957-4484/26/49/495202/meta. [147] Y. Yokomizo and J. Nakamura, Appl. Phys. Lett., 2013, 103, 113901, doi: 10.1063/1.4820820.

[148] K. Yang, Y. Chen, R. D'Agosta, Y. Xie, J. Zhong and A. Rubio, Phys. Rev. B, 2012, 86, 045425, doi: 10.1103/PhysRevB.86.045425.

[149] Zhang, Z., Xie, Y., Peng, Q., and Y. Chen, Sci. Rep., 2016, 6, 21639, doi: 10.1038/srep21639.

[150] Y. Ouyang, Y. Xie, Z. Zhang, Q. Peng and Y. Chen, J. Appl. Phys., 2016, 120, 235109, doi: 10.1063/1.4972831.

[151] W. Oswald W and Z. Wu Energy gaps in graphene nanomeshes Phys. Rev. B, 2012, 85, 115431, doi: 10.1103/PhysRevB.85.115431.

[152] F. Ouyang, S. Peng, Z. Liu, Z. Liu, ACS Nano, 2011, 5, 4023-4030, doi: 10.1021/nn200580w.

[153] M. Vanević, V. M. Stojanović and M. Kindermann, Phys. Rev. B, 2009, 80, 045410, doi: 10.1103/PhysRevB.80.045410T.

[154] G. Pedersen, C. Flindt, J. Pedersen, N. A. Mortensen, A. P. Jauho and K. Pedersen, Phys. Rev. Lett., 2008, 100,136804, doi:10.1103/PhysRevLett.100.136804.

[155] L. Yang, J. Chen, N. Yang and B. Li, Int. J. Heat Mass Transfer, 2015, 91, 428-432, doi: 10.1016/j.ijheatmasstransfer.2015.07.111.

[156] T. Feng, X. Ruan, Carbon, 2016, 101, 107-113, doi: 10.1016/j.carbon.2016.01.082.

[157] T. Gunst, T. Markussen, A. P. Jauho and M. Brandbyge, Phys. Rev. B, 2011, 84, 155449, doi: 10.1103/PhysRevB.84.155449.

[158] H. Karamitaheri, M. Pourfath, R. Faez and H. Kosina, J. Appl. Phys., 2011, 110, 054506, doi: 10.1063/1.3629990.

[159] Y. Yan, Q. F. Liang, H. Zhao, C. Q. Wu and B. Li, Phys. Lett. $\quad$ A, 2012, 376, 2425-2429, doi: 10.1016/j.physleta.2012.06.010.

[160] M. S. Hossain, F. Al-Dirini, F. M. Hossain and E. Skafidas,
Sci. Rep., 2015, 5, 11297, doi: 10.1038/srep11297.

[161] P. H. Chang, M. S. Bahramy, N. Nagaosa, N. and B. K. Nikolic, Nano Lett., 2014, 14, 3779-3784, doi: $10.1021 / \mathrm{nl} 500755 \mathrm{~m}$.

[162] H. Sadeghi, S. Sangtarash and C. J. Lambert, Sci. Rep., 2015, 5, 9514, doi: 10.1038/srep09514.

[163] L. Shao, G. Chen, H. Ye, Y. Wu, H. Niu and Y. Zhu J. Appl. Phys., 2014, 116, 113704, doi: 10.1063/1.4896064.

[164] A. Cupo, P. Masih Das, C. C. Chien, G. Danda, N. Kharche, D. Tristant, M. Drndić and V. Meunier, ACS Nano, 2017, 11, 7494-7507, doi: 10.1021/acsnano.7b04031.

[165] P. Hopkins, L. Phinney, P. Rakich, R. Olsson and I. El-Kady, Appl. Phys. A, 2011, 103, 575-579, doi: 10.1007/s00339-0106189-8.

[166] P. E. Hopkins, P. T. Rakich, R. H. Olsson, I. F. El-Kady, and L. M. Phinney, Appl. Phys. Lett., 2009, 95, 161902, doi: 10.1063/1.3250166.

[167] M. Nomura, J. Shiomi, T. Shiga and R. Anufriev Jpn. J. Appl. Phys., 2018, 57, 080101, doi: 10.7567/JJAP.57.080101/meta.

[168] Q. Hao, Y. Xiao, and Q. Chen, Mater. Today Phys., 2019, 10, 100126, doi: 10.1016/j.mtphys.2019.100126.

[169] Q. Hao and Y. Xiao, Phys. Rev. Appl.,2020,13, 064020, doi: 10.1016/j.mtphys.2019.100126.

[170] B. Y. Cao, W. J. Yao, and Z. Q. Ye, Carbon, 2016, 96, 711719, doi: 10.1016/j.carbon.2015.09.097.

[171] Q. Hao, Y. Xiao, S. Wang, Int. J. Heat Mass Transfer, 2020, 153, 119636, doi: 10.1016/j.ijheatmasstransfer.2020.119636.

[172] M. Yamawaki, M. Ohnishi, S. Ju, J. Shiomi, Sci. Adv., 2018, 4, eaar4192, doi: 10.1126/sciadv.aar4192.

[173] H. Wei, H. Bao and X. Ruan, Nano Energy, 2020, 71, 104619, doi: 10.1016/j.nanoen.2020.104619.

[174] S. Ju, T. Shiga, L. Feng, Z. Hou, K. Tsuda and J. Shiomi, Phys. Rev. X, 2017, 7, 021024, doi: 10.1103/PhysRevX.7.021024.

[175] X. Wan, W. Feng, Y. Wang, H. Wang, X. Zhang, C. Deng, N. Yang, Nano Lett., 2019, 19, 3387-3395, doi: 10.1021/acs.nanolett.8b05196.

[176] T. Wang, C. Zhang, H. Snoussi, G. Zhang, Adv. Func. Mater., 2020, 30, 1906041, doi: 10.1016/j.nanoen.2020.105380.

[177] D. Wu, Z. Yu, J. Xiao, F. Ouyang, F. Phys. E, 2010, 43, $33-$ 39, doi: 10.1016/j.physe.2010.06.017.

[178] X. Liu, Z. Zhang, W. Guo, Small, 2013,9, 1405-1410, doi: 10.1002/smll.201202988.

[179] X. Ji, J. Zhang, Y. Wang, H. Qian, Z. Yu, Nanoscale, 2013, 5, 2527-2531, doi: 10.1039/C3NR33241A.

[180] M. Dvorak, W. Oswald, Z. Wu, Sci. Rep., 2013, 3, 1-7, doi: $10.1038 /$ srep02289.

[181] S. J. Brun, M. R. Thomsen, T. G. Pedersen, J. Phys. Condensed Matter, 2014, 26, 265301, doi: 10.1088/09538984/26/26/265301.

[182] S. Yuan, R. Roldán, A. P. Jauho, M. I. Katsnelson, Phys. Rev. B, 2013, 87, 085430, doi: 10.1103/PhysRevB.87.085430.

[183] J. A. Fürst, J. G. Pedersen, C. Flindt, N. A. Mortensen, M. Brandbyge, T.G. Pedersen and A. P. Jauho, New J. Phys., 2009, 11, 095020, doi: 10.1088/1367-2630/11/9/095020/meta. 
[184] V. Hung Nguyen, M. Chung Nguyen, H.-V. Nguyen, and P. Dollfus, J. Appl. Phys., 2013, 113, 013702, doi: 10.1063/1.4772609.

[185] M. Kim, N. S. Safron, E. Han, M. S. Arnold and P. Gopalan, Nano Lett., 2010, 10, 1125-113, doi: 10.1021/n19032318.

[186] J. Bai, X. Zhong, S. Jiang, Y. Huang, X. Duan, Nat. Nanotechnol., 2010, 5, 190-194, doi: 10.1038/nnano.2010.8.

[187] L. Shi, D. Li, C. Yu, W. Jang, D. Kim, Z. Yao P. Kim A. Majumdar, J. Heat Transfer, 2003, 125, 881-888, doi: 10.1115/1.1597619.

[188] P. Kim, L. Shi, A. Majumdar, P. L. McEuen, Phys. Rev. Lett., 2001, 87, 215502, doi: 10.1103/PhysRevLett.87.215502.

[189] X. Xu, L. F. Pereira, Y., Wang, J. Wu, K. Zhang, X. Zhao, S. Bae, C. T. Bui, R. Xie, J. T. L. Thong, B. H. Hong, K. P. Loh, D. Donadio, B. Li and B. Özylmaz Nat. Commun., 2014, 5, 1-6, doi: $10.1038 /$ ncomms4689.

[190] W. Jang, W. Bao, L. Jing, C. N. Lau, C. Dames, App. Phys. Lett., 2013, 103, 133102, doi: 10.1063/1.4821941.

[191] L. A. Jauregui, Y. Yue, A. N. Sidorov, J. Hu, Q. Yu, G. Lopez, R. Jalilian, D. K. Benjamin, D. A. Delkd and W. Wu, ECS Trans., 2010, 28,73-83, doi:10.1149/1.3367938/meta.

[192] S. Chen, A. L. Moore, W. Cai, J. W. Suk, J. An, C. Mishra, C. Amos, C. W. Magnuson, J. Kang, L. Shi, and R. S. Ruoff, ACS Nano, 2011, 5, 321-328, doi: 10.1021/nn102915x.

[193] S. Sullivan, A. Vallabhaneni, I. Kholmanov, X. Ruan, J. Murthy and L. Shi, Nano Lett., 2017, 17, 2049-2056, doi: 10.1021/acs.nanolett.7b00110.

[194] N. Xiao, X. Dong, L. Song, D. Liu, D. Tay, Y. Wu, L. J. Li, Y. Zhao, T. Yu, H. Zhang, and W. Huang, ACS Nano, 2011, 5, 2749-2755, doi: 10.1021/nn2001849.

[195] J. Oh, H. Yoo, J. Choi, J. Y. Kim, D. S. Lee, M. J. Kim, J. C. Lee, W. N. Kim, J. C. Grossman, J. H. Park and S. S. Lee, Nano Energy, 2017, 35, 26-35, doi: 10.1016/j.nanoen.2017.03.019.

[196] D. Xu, S. Tang, X. Du, Q. Hao, Carbon, 2019, 144, 601607, doi: 10.1016/j.carbon.2018.12.080.

[197] D. Xu, Q. Wang, X. Wu, J. Zhu, H. Zhao, B. Xiao, X. Wang, X. Wang and Q. Hao, Front. Energy, 2018, 12, 127-136, doi: 10.1007/s11708-018-0519-5.

[198] S. Hu, Z. Zhang, P. Jiang, W. Ren, C. Yu, J. Shiomi and J. Chen, Nanoscale, 2019, 11, 11839-11846, doi: 10.1039/C9NR02548K.

[199] F. Liu, Z. Wang, S. Nakanao, S. Ogawa, Y. Morita, M. Schmidt, M. Haque, M. Muruganathan and H. Mizuta, Micromachines, 2020, 11, 387, doi: 10.3390/mi11040387.

[200] H. Wang, K. Kurata, T. Fukunaga, H. Takamatsu, X. Zhang, T. Ikuta, K. Takahashi, T. Nishiyama, H. Ago, and Y. Takata, Sci. Rep., 2016, 6, 1-7, doi: 10.1038/srep21823.

[201] M. S. Kang, S. Y. Kang, W. Y. Lee, N. W. Park, K. C. Kown, S. Choi, G. S. Kim, J. Nam, K. S. Kim, E. Saitoh and H. W. Jang, J. Mater. Chem. A, 2020, 8, 8669-8677, doi: 10.1039/D0TA02629H.

[202] Z. Xiong, X. Wang, K. H. K. Lee, X. Zhan, Y. Chen, and J. Tang, ACS Appl. Mater. Interfaces, 2018, 10, 9211-9215, doi: 10.1021/acsami.8b00097.

[203] Q. Y. Li, X. Zhang and Y. D. Hu, Thermochim. acta, 2014,
592, 67-72, doi: 10.1016/j.tca.2014.08.011.

[204] Q.Y. Li, W. Ma and X. Zhang, Int. J. Heat Mass Transfer, 2016, 95, 956-963, doi: 10.1016/j.ijheatmasstransfer.2015.12.065.

[205] S. Sullivan, A. Vallabhaneni, I. Kholmanov, X. Ruan, J. Murthy, and L. Shi, Nano Lett., 2017, 17, 2049-2056, doi: 10.1021/acs.nanolett.7b00110.

[206] A. K. Vallabhaneni, D. Singh, H. Bao, Murthy and X. Ruan, Phys. Rev. B, 2016, 93, 125432, doi: 10.1103/PhysRevB.93.125432.

[207] Q. Y. Li, T. Feng, W. Okita, Y. Komori, H. Suzuki, T. Kato, T. Kaneko, T. Ikuta, X. Ruan, K. Takahashi, ACS Nano, 2019, 13, 9182-9189, doi: 10.1021/acsnano.9b03521.

[208] Q. Y. Li, K. Takahashi, H. Ago, X. Zhang, T. Ikuta, T. Nishiyama and K. Kawahara, J. Appl. Phys., 2015, 117, 065102, doi: 10.1063/1.4907699.

[209] M. S. Hossain, D. H. Huynh, P. D. Nguyen, L. Jiang, T. C. Nguyen, F. Al-Dirini, F. M. Hossain, E. Skafidas. J. Appl. Phys., 2016, 119, 125106, doi: 10.1063/1.4944710.

[210] M. Y. Han, B. Özyilmaz, Y. Zhang, P. Kim, Phys. Rev. Lett., 2007, 98, 206805, doi: 10.1103/PhysRevLett.98.206805.

[211] M. H. Bae, Z. Li, Z. Aksamija, P. N. Martin, F. Xiong, Z.Y. Ong, I. Knezevic and E. Pop, Nat. commun., 2013, 4, 1-7, doi: 10.1038/ncomms2755.

[212] C. Kamal, M. Ezawa, Phys. Rev. B - Condens. Matter Mater.Phys., 2015, 91,1-10, doi: 10.1103/PhysRevB.91.085423. [213] Q. Y. Li, X. Zhang and K. Takahashi, Int. J. Heat Mass Tran., 2018, 125, 1230-1239, doi: 10.1016/j.ijheatmasstransfer.2018.05.011.

[214] S. Vaziri, E. Yalon M.M. Rojo, S. V. Suryavanshi, H. Zhang, C.J. McClellan, C. S Bailey, K. K. Smithe, A. J. Gabourie, V. Chen, S. Deshmukh, Sci. Adv., 2019, 5, eaax1325, doi: 10.1126/sciadv.aax1325.

[215] C. C. Chen, Z. Li, L. Shi, and S. B. Cronin, Nano Res., 2015, 8, 666-672, doi: 10.1007/s12274-014-0550-8.

[216] P. S. Mahapatra, K. Sarkar, H. R. Krishnamurthy, S. Mukerjee and A. Ghosh, Nano Lett., 2017, 17, 6822-6827, doi: 10.1021/acs.nanolett.7b03097.

[217] N. Poudel, S. Liang, D. Choi, B. Hou, L. Shen, H. Shi, L. K. Ang, L. Shi, and S. Cronin, Sci. Rep., 2017, 7, 1-8, doi: 10.1038/s41598-017-12704-w.

[218] C. C. Chen, Z. Li, L. Shi and S. B. Cronin, Appl. Phys. Lett., 2014, 104, 081908, doi: 10.1063/1.4866335.

[219] J. Oh, Y. Kim, S. Chung, H. Kim, J. G. Son, Adv. Mater. Interfaces, 2019, 6, 1901333, doi:10.1002/admi.201901333.

[220] C. Lee, J. Hong, M. Whangbo and J. H. Shim, Chem. Mater., 2013, 25, 3745-3752, doi: 10.1021/cm402281n.

[221] X. Wang, M. Zebarjadi and K. Esfarjani, Sci. Rep., 2018, 8, 1-9, doi: 10.1038/s41598-018-27430-0.

[222] G. Ding, C. Wang, G. Gao, K. Yao, C. Dun, C. Feng, D. Li and G. Zhang, Nanoscale, 2018, 10, 7077-7084, doi: 10.1039/C7NR09029C.

[223] G. J. Snyder and A. H. Snyder, Energy Environ. Sci., 2017, 10, 2280-2283, doi: 10.1039/C7EE02007D.

[224] J. Wu, Y. Liu, Y. Liu, Y. Cai, Y. Zhao, H.K. Ng, K. 
Watanabe, T. Taniguchi, G. Zhang, C.-W. Qiu, D. Chi, A.H.C. Neto, J.T.L. Thong, K.P. Loh, K. Hippalgaonkar, Proc. Natl. Acad. Sci., 2020, 202007495, doi: 10.1073/pnas.2007495117.

[225] Y. Ge, W. Wan, Y. Ren and Y. Liu, Phys. Rev. Res., 2020, 2, 013134, doi: 10.1103/PhysRevResearch.2.013134.

[226] S. Deng, L. Li, O. J. Guy and Y. Zhang, Phys. Chem. Chem. Phys., 2019, 21, 18161-18169, doi: 10.1039/C9CP03639C.

[227] A. M. Dehkordi, M. Zebarjadi, J. He, T. M. Tritt, Mater. Sci. Eng. Rep., 2015, 97, 1-22, doi: 10.1016/j.mser.2015.08.001.

[228] H. Sadeghi, S. Sangtarash and C. J. Lambert, 2D Mater., 2016, 4, 015012, doi: 10.1088/2053-1583/4/1/015012/meta.

[229] Z. Ding, M. An, S. Mo, X. Yu, Z. Jin, Y. Liao, K. Esfarjani, J. T. Lü, J. Shiomi and N. Yang, J. Mater. Chem. A, 2019, 7, 2114 2121, doi: 10.1039/C8TA10500F.

[230] D. Olaya, M. Hurtado-Morales, D. Gómez, O. CastañedaUribe, Z. Y. Juang and Y. Hernández, 2D Mater., 2017, 5, 011004, doi:10.1088/2053-1583/aa90d8/meta.

[231] P. Jia, Y. Zeng, D. Wu, H. Pan, X. Cao, W. Zhou, Z. X. Xie, J. X. Zhang and K. Q. Chen, J. Phys. Condens. Mat., 2019, 32, 055302, doi: 10.1088/1361-648X/ab4cab/meta.

[232] E. Yalon, C. J. McClellan, K. K. Smithe, M. Muñoz Rojo, R. L. Xu, S. V. Suryavanshi, A. J. Gabourie, C.M. Neumann, F. Xiong, A. B. Farimani and E. Pop, Nano Lett., 2017, 17, 3429 3433, doi: 10.1021/acs.nanolett.7b00252.

[233] J. Hu, T. Wu, J. Tian, N. N. Klimov, D. B. Newell and Y. P. Chen, Nano Energy, 2017, 40, 42-48, doi: 10.1016/j.nanoen.2017.07.035.

[234] X. Wang, M. Zebarjadi and K. Esfarjani, Nanoscale, 2016, 8, 14695-14704, doi: 10.1039/C6NR02436J.

[235] A. Shakouri, J.E. Bowers, Appl. Phys. Lett., 1997, 71, 1234. Doi: 10.1063/1.119861.

[236] G.D. Mahan, J. O. Sofo and M. Bartkowiak, J. Appl. Phys.,1998,8317, doi: 10.1063/1.367255.

[237] G. D. Mahan, J. Appl. Phys., 1994, 76 4362-4366, doi: 10.1063/1.357324.

[238] G. Fitzpatrick, E. Britt and B. Moyzhes, Proc. ThirtySecond Intersoc. Energy Convers. Eng. Conf. (Cat. No.97CH6203), IEEE, 1997, 2, 1045-1051, doi: 10.1109/IECEC.1997.661913.

[239] H. MOSS, J. Electron. Control., 1957, 2, 305-322, doi: 10.1080/00207215708937036.

[240] K. G. Hernqvist, M. Kanefsky and F. H. Norman, RCA. Rev., 1958, 19, 244-258.

[241] Thermionic energy conversion. In Nanoscale Energy Transp., M.G. Rosul, M.S. Akhanda, M. Zebarjadi, IOP Publishing, 2020; pp. 14-1-14-29, doi: 10.1088/978-0-75031738-2ch14.

[242] M. Zebarjadi, Solid-State Thermionic Power Generators: An Analytical Analysis in the Nonlinear Regime, Phys. Rev. Appl., 2017, 8, 014008, doi: 10.1103/PhysRevApplied.8.014008.

[243] X. Wang, M. Zebarjadi and K. Esfarjani, Sci. Rep., 2018, 8, 1-9, doi: 10.1038/s41598-018-27430-0.

[244] S.-J. Liang, B. Liu, W. Hu, K. Zhou and L.K. Ang, Sci. Rep., 2017, 7, 46211, doi: 10.1038/srep46211.

[245] H. Sadeghi, S. Sangtarash, C.J. Lambert, 2D Mater., 2016,
4, 015012, doi:10.1088/2053-1583/4/1/015012.

[246] D.G. Cahill, Rev. Sci. Instrum., 2004, 75, 5119-5122, doi: 10.1063/1.1819431.

[247] K.M. Freedy, T. Zhu, D.H. Olson, P.M. Litwin, P. Hopkins, M. Zebarjadi and S.J. McDonnell, 2D Mater, 2020, 7, doi: 10.1088/2053-1583/ab834b.

[248] M.G. Rosul, D. Lee, D.H. Olson, N. Liu, X. Wang, P.E. Hopkins, K. Lee and M. Zebarjadi, Sci. Adv., 2019, 5, eaax7827, doi: 10.1126/sciadv.aax7827.

[249] J. Lim, H. T. Wang, J. Tang, S. C. Andrews, H. So, J. Lee, D. H. Lee, T.P. Russell, and P. Yang, ACS Nano, 2016, 10, 24-132, doi: 10.1021/acsnano.5b05385.

[250] J. Mahmood, E.K. Lee, M. Jung, D. Shin, I.Y. Jeon, S.M. Jung, H. J. Choi, J. M. Seo, S.Y. Bae, S.D. Sohn, and N. Park, Nat. Commun., 2015,6,1-7, doi: 10.1038/ncomms7486.

[251] J. Mahmood, E. K. Lee, M. Jung, D. Shin, H.J. Choi, J. M. Seo, S. M. Jung, D. Kim, F. Li, M.S. Lah, N. Park, Proc. National Academy Sci., 2016, 113, 7414-7419, doi: 10.1073/pnas.1605318113.

[252] M. D. Fischbein and M. Drndić, Appl. Phys.Lett., 2008, 93, 113107, doi: 10.1063/1.2980518.

[253] C. J. Russo and J. A. Golovchenko, Pro. National Academy Sci., 2012, 109, 5953-5957, doi:10.1073/pnas.1119827109.

[254] O. Dyck, M. Ziatdinov, D. B. Lingerfelt, R.R. Unocic, B. M. Hudak, A. R. Lupini, S. Jesse and S. V. Kalinin, Nature Rev. Mater, 2019, 4, 497-507, doi: 10.1038/s41578-019-0118-z.

[255] S. Fujii, T. Yokoi, C. A. Fisher, H. Moriwake and M. Yoshiya, Nat. Commun., 2020, 11, 1-10, doi: 10.1038/s41467020-15619-9.

[256] H. Zhang, K. Hippalgaonkar, T. Buonassisi, O. M. Løvvik, E. Sagvolden, D. Ding, ES Energy Environ., 2018, 2, 1-8, doi: 10.30919/esee8c191.

[257] L. Wu, Y. Xiao, M. Ghosh, Q. Zhou, Q. Hao, ES Mater. Manuf., 2020, 9, 3, doi: 10.30919/esmm5f756.

[258] T. Mueller, A. Hernandez and C. Wang, J. Chem. Phys., 2020, 152, 050902, doi:10.1063/1.5126336.

[259] B. Mortazavi, E. V. Podryabinkin, S. Roche, T. Rabczuk, X. Zhuang and A. V. Shapeev, Mater. Horiz., 2020, 7, 2359-2367, doi:10.1039/D0MH00787K.

[260] X. Qian, S. Peng, X. Li, Y. Wei, R. Yang, Mater. Today Phys., 2019, 10, 100140, doi: 10.1016/j.mtphys.2019.100140.

[261] A. Harzheim, J. Spiece, C. Evangeli, E. McCann, V. Falko, Y. Sheng, J. Warner, G. Briggs, J. Mol, P. Gehring, and O. Kolosov, Nano Lett. 2018, 18, 7719-7725, doi: 10.1021/acs.nanolett.8b03406.

[262] A. Harzheim, F. Könemann, B. Gotsmann, H.v.d. Zant, P. Gehring, Adv. Func. Mater., 2020, 30, 2000574, doi: 10.1002/adfm.202000574. 


\section{Author information}
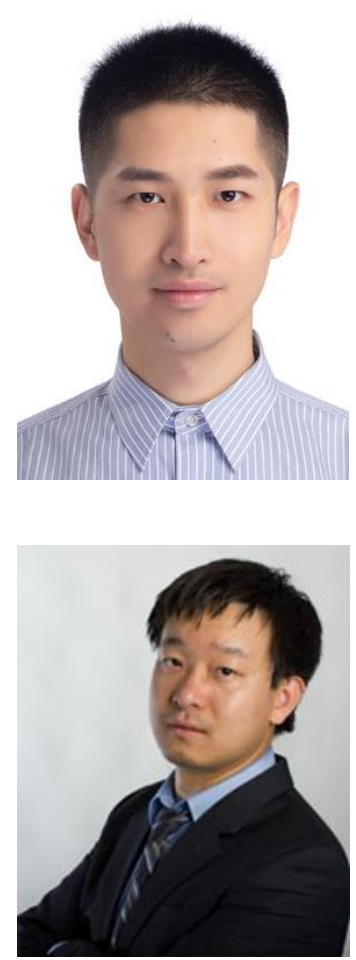

Qinyi Li obtained his B. Eng. and Dr. Eng. from Tsinghua University, in 2011 and 2016, respectively. After that, he joined Kyushu University and has been working as an assistant professor. His research interests are focused on nanoscale heat and mass transfer, thermoelectric energy conversion, and other clean energy technologies.

Qing Hao is an Associate Professor of Department of Aerospace and Mechanical Engineering at the University of Arizona. His research focuses on nanoscale energy transport in materials and electronic devices, including thermoelectrics, highpower electronics, and 2D materials. He received the 2008 R\&D 100 Award as a team member for thermoelectrics research, 2015 AFOSR YIP Award for graphene studies, and 2017 NSF CAREER Award for thermal studies of grain boundaries. He was selected as the inaugural class of the Craig M. Berge Dean's Fellows in 2020.

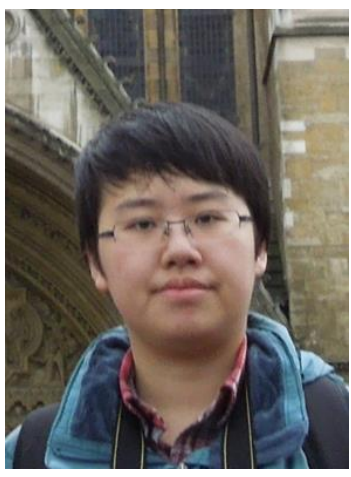

Tianhui Zhu is currently a Ph.D. candidate in the Department of Electrical and Computer Engineering at University of Virginia. She obtained B.S. and M.Phil. degrees in Physics from University of Manchester in UK and obtained M.S. in Physics from Rutgers University prior to joining University of Virginia. Her research interests include thermoelectric transport in 2D materials and thin films.

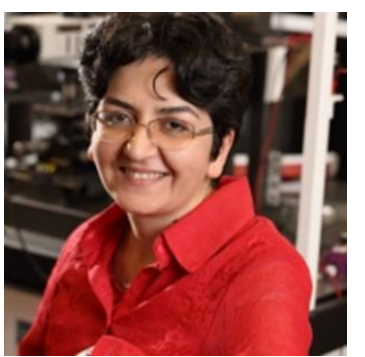

Mona Zebarjadi is a joint professor of Electrical and Computer Engineering and Materials Science and Engineering Departments at University of Virginia. Prior to her current appointment she was a professor of Mechanical Engineering Department at Rutgers University. Her research interests are in electron and phonon transport modeling; materials and device design, fabrication and characterization; with emphasis on energy conversion systems such as thermoelectric, thermionic, and thermomagnetic power generators, and heat management in high power electronics and optoelectronic devices. She received her Bachelor's and master's degree in physics from Sharif University and her PhD in EE from UCSC in 2009, after which she spent 3 years at MIT as a postdoctoral fellow working jointly with electrical and mechanical engineering departments. She is the recipient of 2017 NSF Career award, 2014 AFOSR career award, 2015 A.W. Tyson assistant professorship award, MRS graduate student gold medal, and SWE electronics for imaging scholarship.

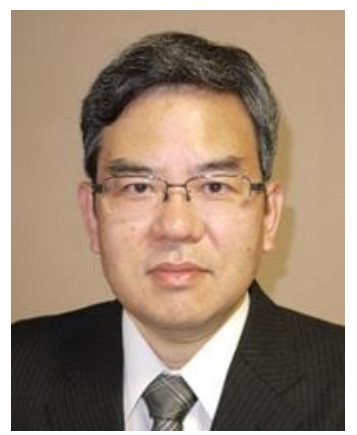

K. Takahashi obtained his B. Eng. and Dr. Eng. from the University of Tokyo, in 1984 and 1992, respectively. After that, he joined Kyushu University and has been working as a full professor since 2010. His current research interests are focused on nanoscale heat transfer and nanofluidics.

Publisher's Note: Engineered Science Publisher remains neutral with regard to jurisdictional claims in published maps and institutional affiliations. 